Article

\title{
Comparative Analysis and Optimization of Power Loss Based on the Isolated Series/Multi Resonant Three-Port Bidirectional DC-DC Converter
}

\author{
Bo Chen, Ping Wang, Yifeng Wang * (D), Wei Li, Fuqiang Han and Shuhuai Zhang
}

School of Electrical Engineering and Automation, Tianjin University, Tianjin 300072, China; Cb92614@126.com (B.C.); pingw@tju.edu.cn (P.W.); liweitju@hotmail.com (W.L.); fqhanyz@foxmail.com (F.H.); zhangshuhuai@tju.edu.cn (S.Z.)

* Correspondence: wayif@tju.edu.cn; Tel.: +86-022-2740-6007; Fax: +86-022-2740-1479

Received: 3 September 2017; Accepted: 6 October 2017; Published: 11 October 2017

\begin{abstract}
Based on the loss distribution and efficiency analysis, a comparative study between a series resonant three-port bidirectional DC-DC converter (SR-TBC) and a multi-resonant three-port bi-directional DC-DC converter (MR-TBC) is reported here. By using the Fourier equivalent analysis method in hand, the resonant current, switching current expressions, zero voltage soft switching (ZVS) conditions of MR-TBC and SR-TBC are deduced. Besides, in consideration of efficiency and soft switching aspects, the loss models of main power components and resonant elements are integrated and optimized for both topologies. Their loss distributions are established, and the different effects derived from the adoption of SiC MOSFET and Si MOSFET on the converter efficiency are discussed. Finally, to verify the theoretical analyses, comparative experiments under diverse load states are conducted based on the prototypes of the MR-TBC and SR-TBC. The obtained results demonstrate that the MR-TBC successfully broadens the ZVS range and thus achieves higher efficiency along the entire load range.
\end{abstract}

Keywords: bi-directional DC-DC converter; three-port converter; soft switching; multi-resonant converter; loss distribution; SiC MOSFET

\section{Introduction}

Nowadays, as the World is facing the increasingly serious energy crisis and pollution problems, more and more attention is being paid to the development and utilization of renewable energy sources [1-3]. However, the output characteristics of renewable energy sources, such as solar energy and wind energy, are extremely unstable due to their inherent features of time variance and intermittency. Therefore, as a part of the distributed generation system (DEGS), the energy storage system plays a vital role in improving its output stability and increasing the energy utilization efficiency [4-6]. In the existing DEGS, the renewable energy sources, storage units and loads are connected together by multiple independent converters, as Figure 1 shows [7]. However, the use of a large number of independent converters increases dramatically the complexity of the DEGS system, and the necessary sophisticated control procedures also lower the overall operating performance and total efficiency. In order to solve these problems, three-port bidirectional DC-DC converters (TP-BDCs) were developed, which not only simplify the overall complexity of the system, but improve the power density as well. Therefore, their fruitful advantages make TP-BDCs competitive in a wide range of applications [8-24]. 


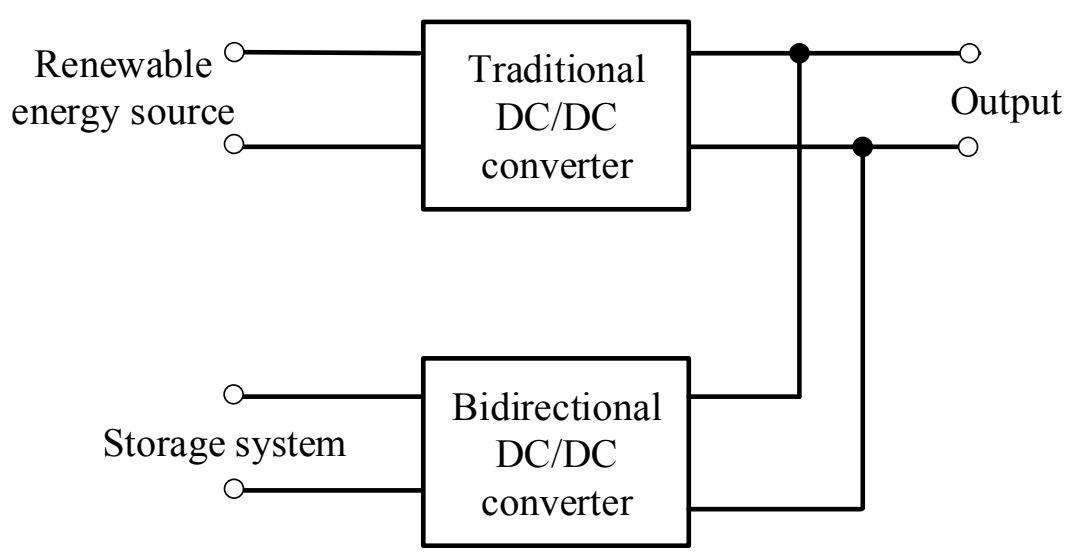

(a)

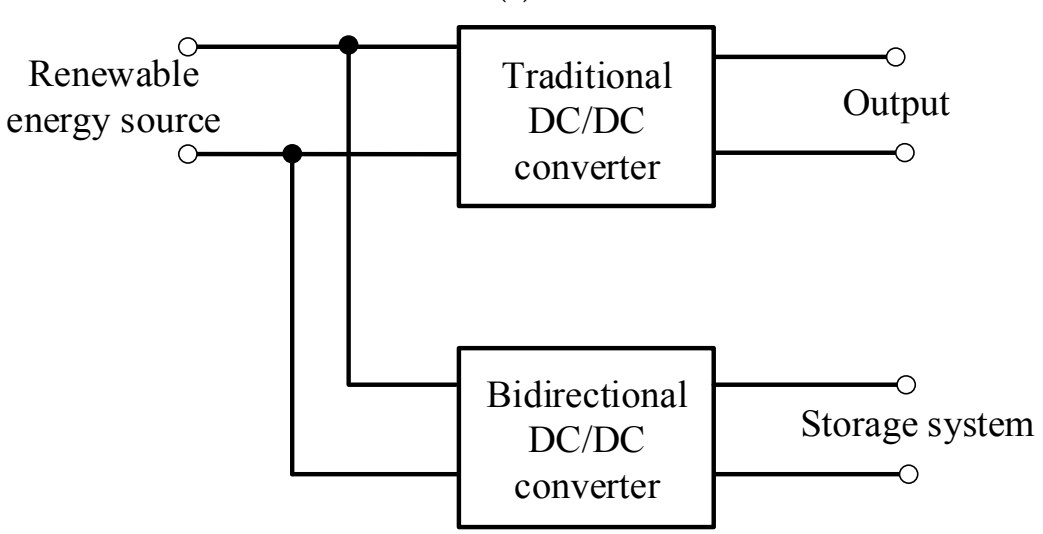

(b)

Figure 1. The structures of traditional DEGS (a) type 1; (b) type 2.

According to the connection mode, TP-BDC topologies can be divided into three types: the nonisolated TP-BDC, the partly isolated TP-BDC and the isolated TP-BDC. Among them, the non-isolated type is beneficial due to the fewer number of components and compact structure, while it limits the voltage gain as the price $[8,9]$. The partly isolated TP-BDC can overcome this issue by using a transformer which can isolate one port from the remaining ports [10-12]. Unfortunately, both the non-isolated type and the partly isolated type are still not suitable for applications where the completely isolated characteristic is the most important requirement for all ports, therefore the isolated TP-BDC must also be investigated $[13,14]$.

The isolated TP-BDC exhibits superiority over its two counterparts, because three-port power exchange with complete galvanic isolation can be realized by using a three-winding transformer. The device has become a research hotspot by virtue of its high efficiency, high reliability, and complete galvanic isolation. Then, with further studies in this field, a sub-classification has been proposed among the numerous published references [15-24], and two typical structures are proposed, namely the triple-active-bridge structure (TAB), and the resonant three-port bidirectional DC-DC converter, as shown in Figure 2.

In [15-18], the TAB as shown in Figure 2a is described. Besides the inherent characteristics of complete galvanic isolation, the TAB also shares the ZVS characteristics and bidirectional operation by using the phase shift control method, but it is only suitable for low frequency applications since the power exchange is inversely proportional to the impedance $L_{1}, L_{2}$ and $L_{3}$ including the leakage inductance of the transformer. Therefore, the working frequency must be reduced under high power conditions due to parameter limitations $[17,19]$. This is also troublesome for the realization of high power density. 
Another type of structure, the series-resonant three-port bidirectional DC-DC converter (SR-TBC), shown in Figure 2b, was first proposed in [20-22]. This topology not only retains all the advantages of $\mathrm{TAB}$, but can achieve the desirable high power density by simply promoting the operating frequency because the impedance of SR-TBC can be adjusted by the series-resonant tanks $C_{R 1}, L_{R 1}, C_{R 2}$ and $L_{R 2}$. Furthermore, the resonant frequency is also defined by the parameters of $C_{R 1}, L_{R 1}, C_{R 2}$ and $L_{R 2}$.

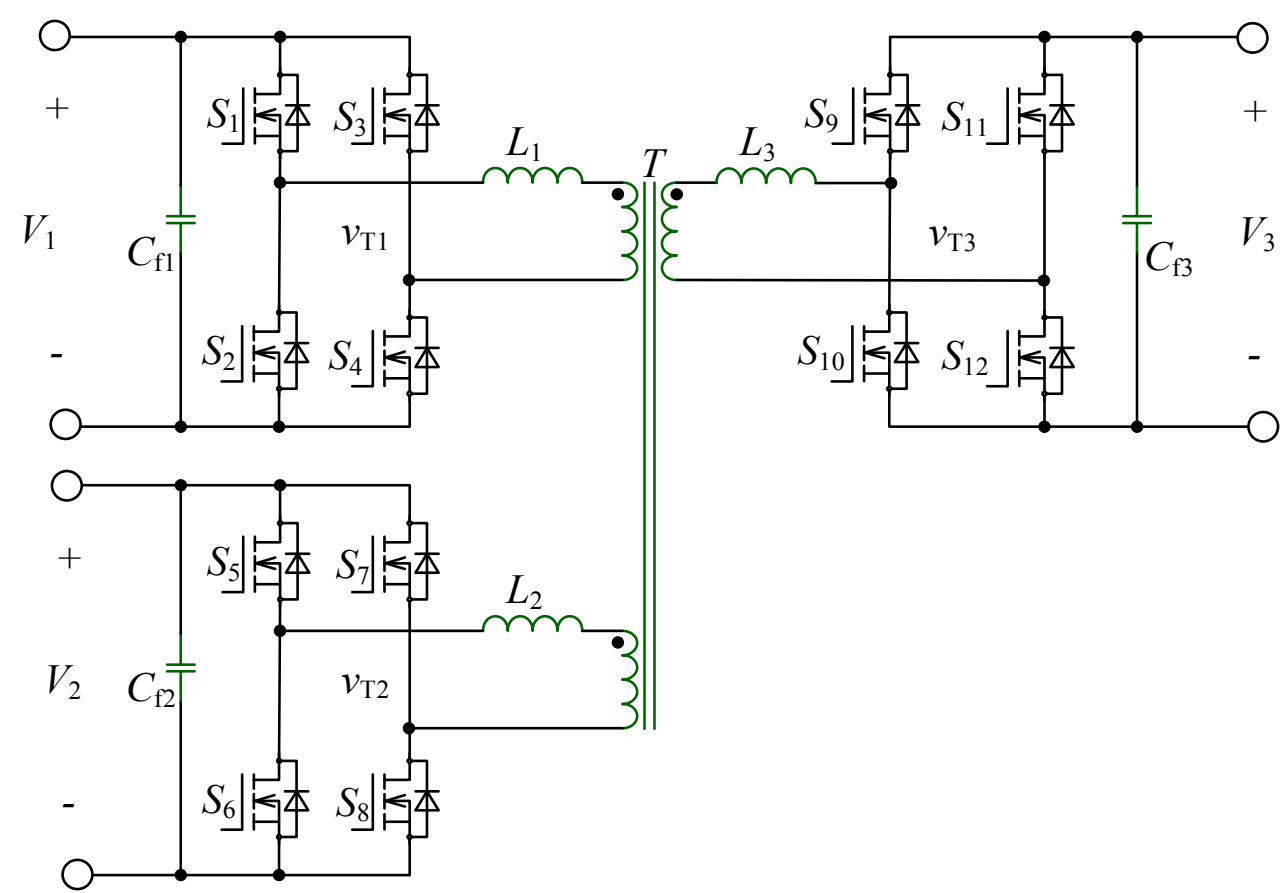

(a)

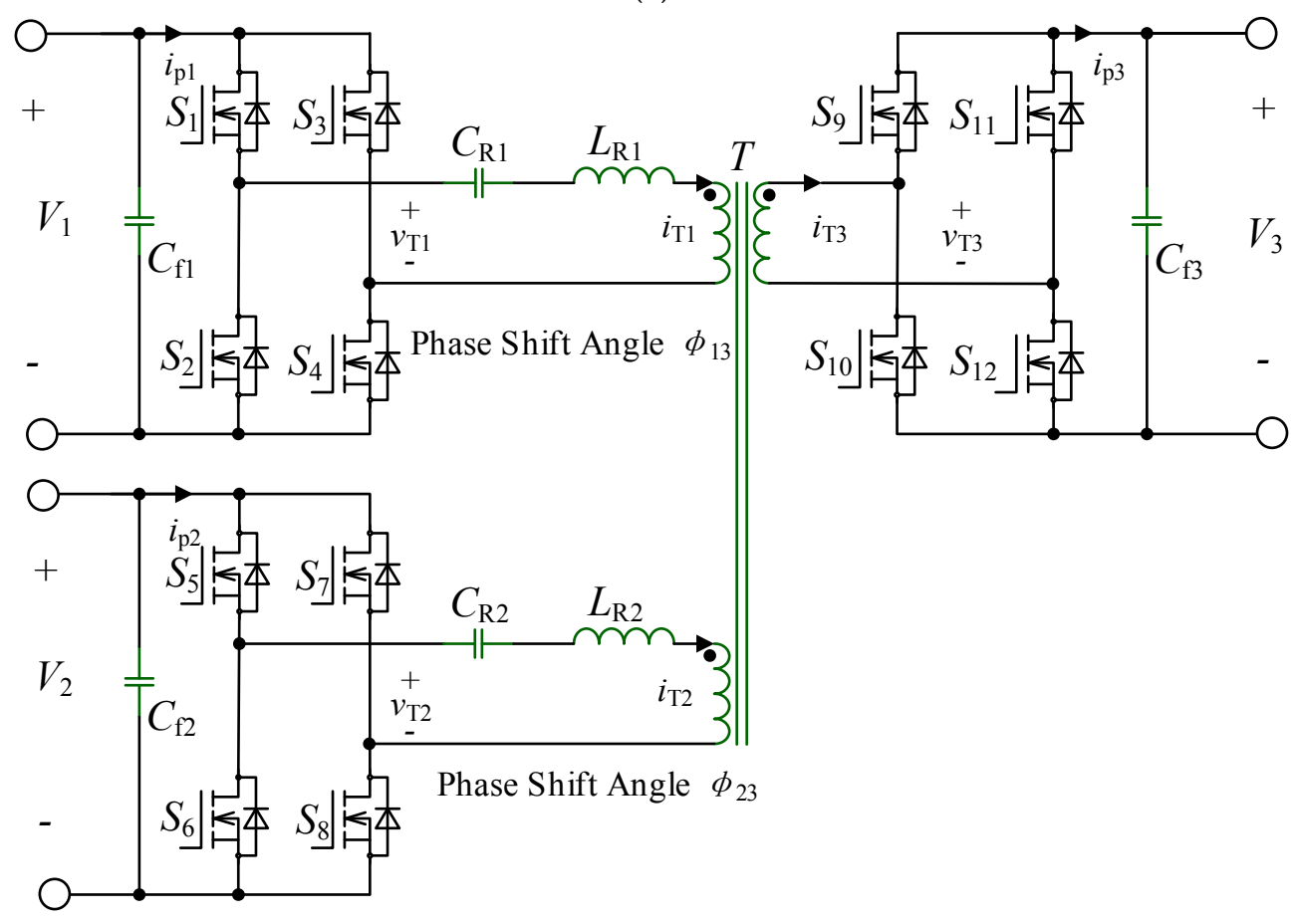

(b)

Figure 2. Cont. 


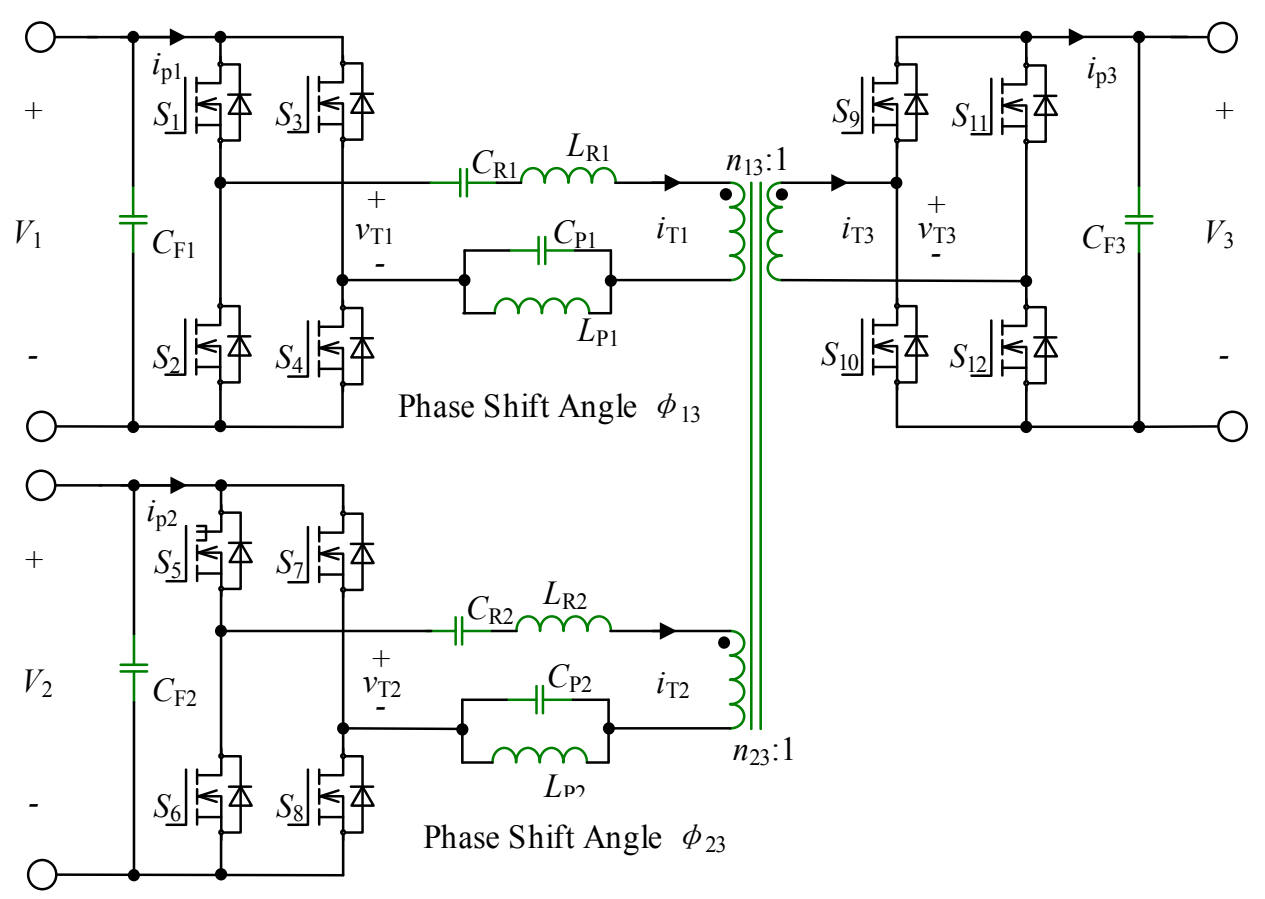

(c)

Figure 2. The structures of TP-BDC (a) TAB; (b) SR-TBC; (c) MR-TBC.

Thus, it can achieve integrated management of switch frequency and impedance, and can work with a higher switching frequency in the case of high power levels. Unfortunately, this converter suffers from progressive efficiency reduction under light loads. This structure is further expanded in [23]. By introducing the $L C L C$ resonant structure, the multi-resonant three port bidirectional DC-DC converter (MR-TBC) is proposed, as shown in Figure 2c. Compared to the SR-TBC, MR-TBC has more resonant components, while the power transmission of the 3rd harmonic component is realized as a trade-off. Besides, by introducing the 3rd harmonic component of the current, the MR-TBC would reduce the peak current and realizes ZVS for all switches, thus ensuring high efficiency. However, in [23], the operational characteristics of the topology are analyzed and verified only in theory. The efficiency and the distribution of losses under different loads are not explained in detail. Also, detailed comparisons between SR-TBC and MR-TBC in terms of loss analysis, efficiency, and load are lacking further discussion, although the relevant contents are important to analyze and optimize the characteristics of the converter.

For power converters, especially for the high frequency applications, the loss analysis is crucial due to its guiding significance for the efficiency estimation and magnetic design [24-30]. Therefore, an increasing number of scholars have focused their attention on the loss analysis for various converters. In [24], the loss distribution of various components was investigated in detail, and both the power switching devices and the passive components were studied. In $[25,26]$, the switch and diode loss are studied and compared in detail for several converter topologies, and the specific loss distribution of the corresponding topology is also given. In [27], the losses of diodes with various materials is compared, and the loss distribution based on the corresponding topology is also given. Although comprehensive investigations have been conducted in these references, their proposed methods are only applicable to low-frequency alternatives. For high frequency applications, the poor accuracy makes these methods awkward to apply. The key reason that leads to this phenomenon is attributable to the fact that some losses, often ignored in low frequency situations, become a noticeable factor of the total loss in high frequency operation conditions. On the other hand, with the increase of frequency, the selection of switching devices is another significant factor for overall power converter efficiency. With the development of SiC devices, more and more applications have begun to use these devices 
because of their inherent characteristics [28-30]. However, the specific scenario of the loss analysis and comparison of $\mathrm{SiC}$ devices and $\mathrm{Si}$ devices is still a barren research field. Last but not least, load also displays a great influence on the loss distribution of the converter. Therefore, under different loads, the loss analysis is of great significance for the three ports resonant DC-DC converter to improve efficiency and realize high frequency, but this aspect is rarely reported.

In this paper, a systematic loss analysis method is developed for the MR-TBC and the SR-TBC. Comparisons of loss distributions between the MR-TBC and the SR-TBC are implemented under different loads. The advantages and disadvantages of the two topologies are analyzed under different working conditions. The corresponding prototype and experiments are established to verify the accuracy of theoretical analysis. The article is organized as follows: in Section 2, the operating modes of MR-TBC and SR-TBC are briefly described. In Section 3, a detailed analysis of the steady-state operation characteristics of the two topologies is carried out. In Section 4, the corresponding loss model is established for the converters. In Section 5, the comparison of the loss distribution between SR-TBC and MR-TBC are analyzed by theoretical calculations, and some device optimizations are also given. In Section 6, the relevant experiments are implemented to verify the correctness of the theory and the accuracy of the calculation. In the last section, relevant conclusions are summarized.

\section{Operating Principle of the MR-TBC and SR-TBC}

In this section, the main current and voltage waveforms under the operating modes of MR-TBC and SR-TBC are given, as Figure 3 shows. In the picture, $v_{\mathrm{G}_{-} \mathrm{S} x}$ means the gate voltage of switch $\mathrm{S}_{x}$ $(x$ is $1-12) ; v_{\mathrm{T} 1}-v_{\mathrm{T} 3}$ are defined as the three-port voltages of the transformer, respectively; $i_{\mathrm{T} 1}-i_{\mathrm{T} 3}$ also represents the corresponding port currents of the transformer; $i_{\mathrm{P} 1}-i_{\mathrm{P} 3}$ are the corresponding full bridge current of the three ports. All these variables can be found in Figure 2b,c.

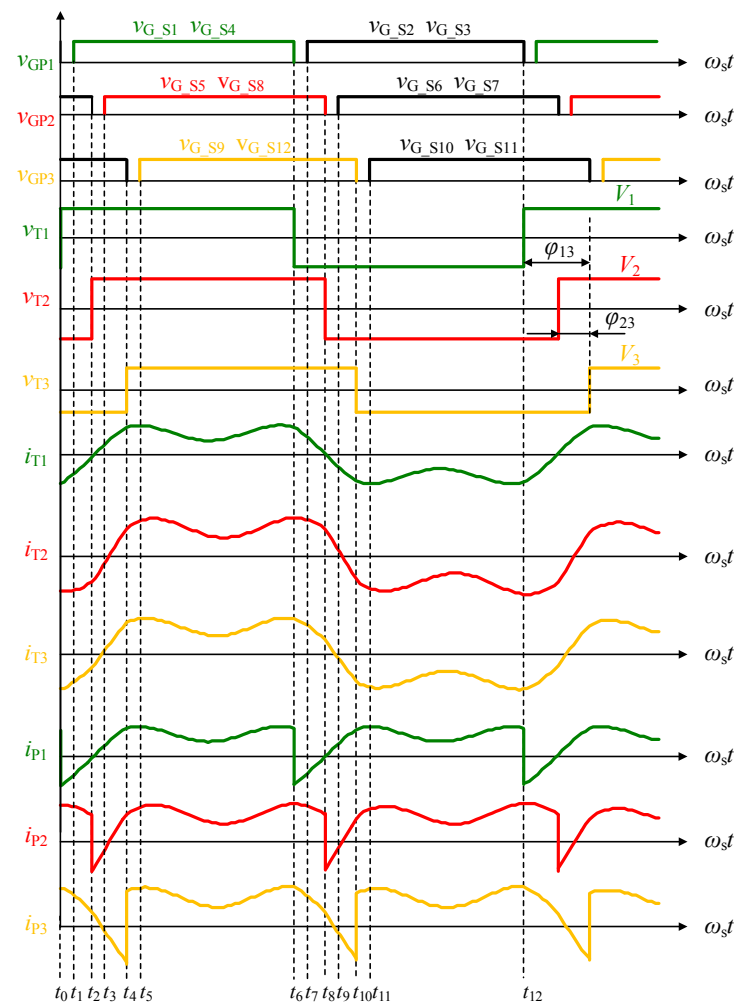

(a)

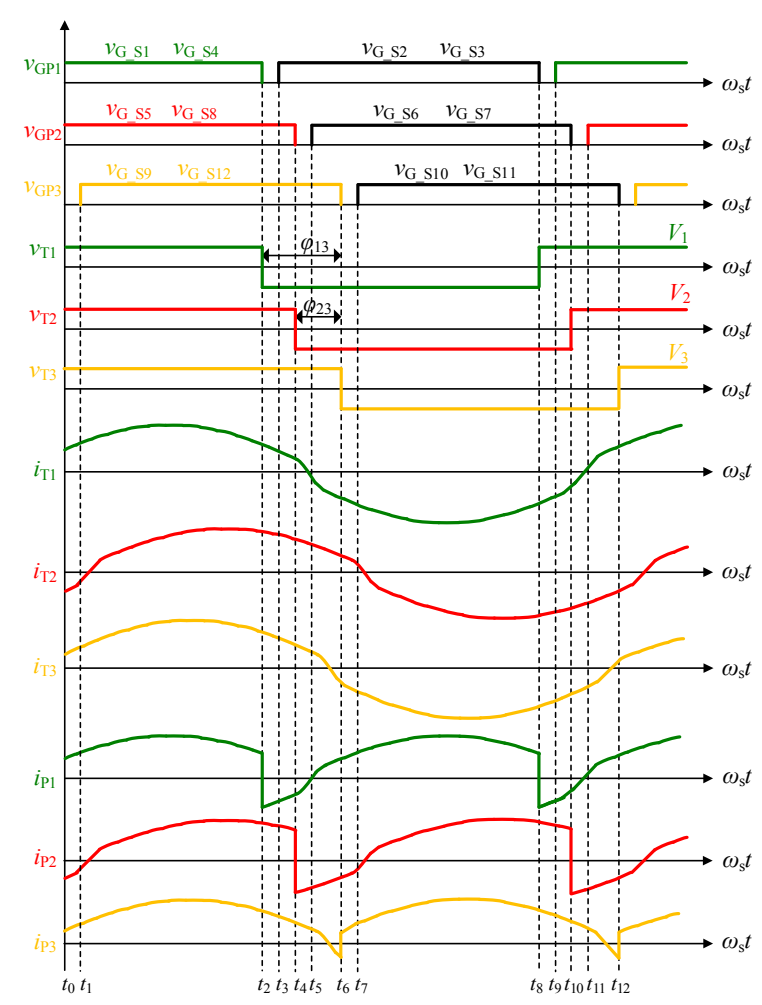

(b)

Figure 3. The current and voltage waveforms of MR-TBC and SR-TBC; (a) MR-TBC; (b) SR-TBC. 
Due to article length limitations, only the main current and voltage waveforms of MR-TBC and SR-TBC for a certain case are presented. The basic operating modes of the MR-TBC and the SR-TBC can be understood based on these major current and voltage waveforms. Interested readers can find a detail operational modal analysis of MR-TBC in [23]; similarly, a detailed operational modal analysis of SR-TBC is presented in [21,22].

\section{Steady State Analysis}

In this section, a steady-state analysis is conducted for the SR-TBC and MR-TBC separately, including the expressions of the three ports resonant current, transformer port voltages and the turn-off currents of switches in the three ports. Especially, the turn-off currents are of great significance for the loss distribution analysis. As Figure 2c shows, the three port voltages $V_{1}, V_{2}$ and $V_{3}$ correspond to the voltage of battery $V_{\mathrm{BAT}}$, voltage of supercapacitors $V_{\mathrm{SC}}$ and bus voltage $V_{\mathrm{BUS}}$; the three-port powers are $P_{1}, P_{2}$ and $P_{3}$; the three-port transformer currents are $i_{\mathrm{T} 1}, i_{\mathrm{T} 2}$ and $i_{\mathrm{T} 3}$. The turns ratio of the transformer is defined as $n_{13}: n_{23}: 1$. The output voltage of the three rectifier bridges are respectively $v_{\mathrm{T} 1}, v_{\mathrm{T} 2}$ and $v_{\mathrm{T} 3}$. The phase shift angle between ports 1 and 3 is defined as $\varphi_{13}$, and the corresponding phase shift angle between ports 2 and 3 is $\varphi_{23}$. When $v_{\mathrm{T} 1}$ and $v_{\mathrm{T} 2}$ lead $v_{\mathrm{T} 3}, \varphi_{13}$ and $\varphi_{23}$ are defined as positive. As the MR-TBC and SR-TBC are both bidirectional converters, the positive direction is defined as the power transfer from ports 1 and 2 to port 3 (ports 1 and 2 work as input; port 3 works as output).

As shown in Figure 4, the resonant cavity of SR-TBC is constituted by a capacitor $C_{\mathrm{r}}$ and an inductance $L_{R}$, and thus it only possesses one frequency $f_{r}$; while, the resonant tank of the MR-TBC is composed of four elements including $C_{\mathrm{r}}, L_{\mathrm{R}}, C_{\mathrm{P}}$ and $L_{\mathrm{P}}$, resulting in more resonant frequencies $f_{\mathrm{r} 1}, f_{\mathrm{r} 2}$ and $f_{\mathrm{r} 3}$. The parameters of resonant elements must be designed appropriately to meet the requirements $f_{\mathrm{r} 2}=2 f_{\mathrm{r}}, f_{\mathrm{r} 3}=3 f_{\mathrm{r}}$. In this way, both 1st and 3rd harmonic power can be transferred through the resonant tank. Besides, owing to the superposition of the two order harmonic powers, the resonant current presents the saddle-shaped waveform as shown in Figure 5 . In this figure, $v_{\mathrm{SRT}}$ and $i_{\mathrm{SRT}}$ represent the resonant current and the voltage of resonant cavity for SR-TBC; similarly, $v_{\mathrm{MRT}}$ and $i_{\mathrm{MRT}}$ are the resonant current and the voltage of resonant cavity for MR-TBC.

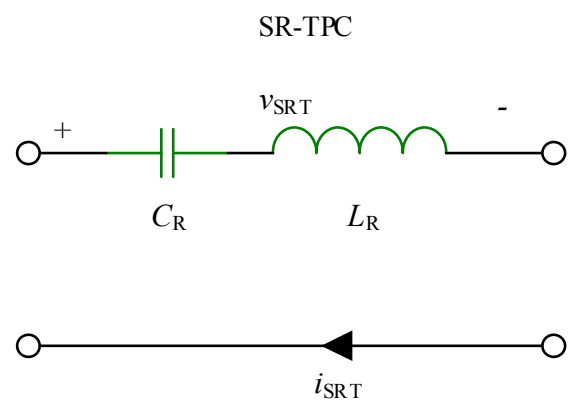

(a)

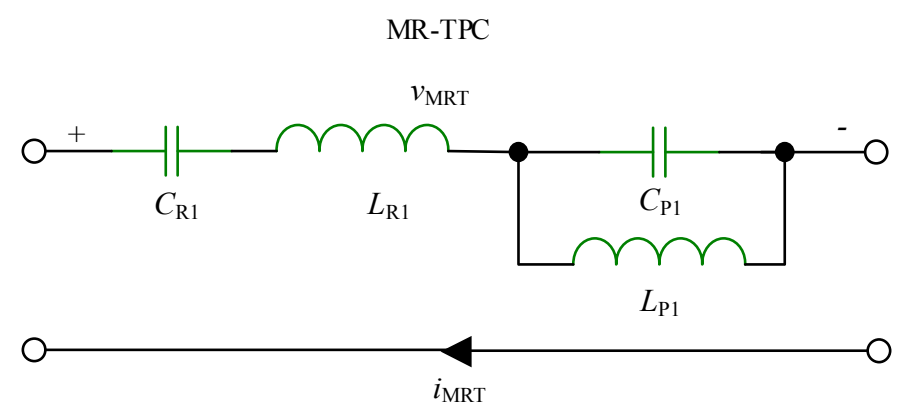

(b)

Figure 4. The comparison of resonant cavity structures (a) SR-TBC; (b) MR-TBC.

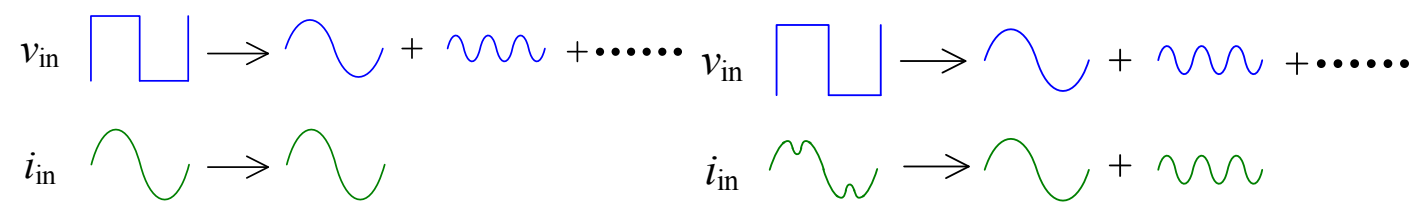

(a)

(b)

Figure 5. The comparison of resonant cavity current and voltage (a) SR-TBC; (b) MR-TBC. 
Compared to SR-TBC, the 3rd frequency component is introduced in MR-TBC, so that the power circulation is reduced, which results from the high frequency component of the square wave voltage and the resonant current. Thus, MR-TBC can reduce the loss of conduction, contributing to higher efficiency. In addition, due to the superposition of 3rd frequency component, the peak value of the resonant current has been effectively reduced, which can reduce current stress. At the same time, the ZVS range is widened by MR-TBC.

However, the traditional time-domain analysis method is extremely complex and needs a great amount of calculation work, due to the multiple resonant elements and operating modes. In this paper, the Fourier equivalent method is used and the square wave voltages of the output are approximately equivalent to the sum of the 1st and 3rd harmonic components. By this method, the calculation is simplified, while the accuracy of the calculation results can also be guaranteed. Similarly, in order to facilitate the design of parameters, a per-unit model for converter is developed. All the equations presented are normalized on the basis of Equation (1):

$$
V_{\mathrm{B}}=V_{3} \quad P_{\mathrm{B}}=P_{3} \quad Z_{\mathrm{B}}=V_{3}^{2} / P_{\mathrm{B}} \quad I_{\mathrm{B}}=V_{\mathrm{B}} / Z_{\mathrm{B}}
$$

where $V_{3}$ is the voltage of BUS side; $P_{3}$ is the power of BUS side; In addition, The normalized driving frequency is defined as: $F=f_{\mathrm{sw}} / f_{\mathrm{r}}$.

In order to facilitate the phase-shifting control, the resonant cavities of SC and BAT use the same design parameters. For convenience, the $C_{\mathrm{R} 1}$ and $L_{\mathrm{R} 1}$ are defined as the series resonant capacitor and inductor, while $C_{\mathrm{p} 1}$ and $L_{\mathrm{p} 1}$ are defined as parallel resonant cavity capacitance and inductance. Thus, the input impedances at three resonant frequencies can be deduced as Equation (2) for the resonant cavity:

$$
\left\{\begin{array}{l}
X_{\mathrm{r} 1}=\omega_{\mathrm{r}} L_{\mathrm{R} 1}-\frac{1}{\omega_{\mathrm{r}} C_{\mathrm{R} 1}}+\frac{\omega_{\mathrm{r}} L_{\mathrm{P} 1}}{1-\omega_{\mathrm{r}}^{2} L_{\mathrm{P} 1} C_{\mathrm{P} 1}}=0 \\
X_{\mathrm{r} 2}=2 \omega_{\mathrm{r}} L_{\mathrm{R} 1}-\frac{1}{2 \omega_{\mathrm{r}} C_{\mathrm{R} 1}}+\frac{2 \omega_{\mathrm{r}} L_{\mathrm{P}}}{1-4 \omega_{\mathrm{r}} L_{\mathrm{P}} C_{\mathrm{P} 1}}=\infty \\
X_{\mathrm{r} 3}=3 \omega_{\mathrm{r}} L_{\mathrm{R} 1}-\frac{1}{3 \omega_{\mathrm{r}} C_{\mathrm{R} 1}}+\frac{3 \omega_{\mathrm{r}} L_{1}}{1-9 \omega_{\mathrm{r}}^{2} L_{\mathrm{P} 1} C_{\mathrm{P} 1}}=0
\end{array}\right.
$$

where $\omega_{\mathrm{s}}$ and $\omega_{\mathrm{r}}$ are the corresponding angular velocities of $f_{\mathrm{s}}$ and $f_{\mathrm{r}}$ respectively. Then, the proportional relationship between resonant elements can be deduced as:

$$
C_{\mathrm{R} 1}=\frac{5}{3} C_{\mathrm{P} 1}, L_{\mathrm{R} 1}=\frac{16}{15} L_{\mathrm{P} 1}, 4 \omega_{\mathrm{r}}^{2}=C_{\mathrm{P} 1} L_{\mathrm{P} 1}
$$

Base on Equation (3), the impedance of the resonant cavity under angular velocities can be deduced as:

$$
X=\frac{16}{15} \omega L_{\mathrm{P} 1}-\frac{12 \omega_{\mathrm{r}}^{2} L_{\mathrm{P} 1}}{5 \omega}+\frac{4 \omega_{\mathrm{r}}^{2} \omega L_{\mathrm{P} 1}}{4 \omega_{\mathrm{r}}^{2}-\omega^{2}}
$$

where $\omega$ represents angular velocity. Combining (1)-(4), for a multi-resonant cavity, the impedance at 1st and 3rd harmonic frequency can be written as:

$$
\left\{\begin{array}{c}
X_{\mathrm{s} 1, \mathrm{pu}}=Q\left(\frac{16}{15} F-\frac{12}{5 F}+\frac{4 F}{4-F^{2}}\right) \\
X_{\mathrm{s} 3, \mathrm{pu}}=Q\left(\frac{16}{5} F-\frac{4}{5 F}+\frac{12 F}{4-9 F^{2}}\right)
\end{array}, Q=\frac{\omega_{\mathrm{r}} L_{\mathrm{P} 1}}{Z_{\mathrm{B}}}\right.
$$

For the series resonant capacitor $C_{r}$, its impedance at 1st and 3rd harmonic frequency can be written as:

$$
\left\{\begin{array}{l}
X_{\mathrm{CR} 1, \mathrm{pu}}=\frac{1}{\omega_{\mathrm{s}} C_{\mathrm{R} 1} Z_{\mathrm{B}}} \\
X_{\mathrm{CR} 3, \mathrm{pu}}=\frac{1}{3 \omega_{\mathrm{S}} C_{\mathrm{R} 1} Z_{\mathrm{B}}}
\end{array}\right.
$$


The transformer is treated as an ideal model. The conduction resistances of switches and some equivalent series resistances in other devices are ignored. In this case, according to the Fourier equivalent method, the three port voltages are expressed as:

$$
\left\{\begin{array}{c}
v_{\mathrm{T} 1, \mathrm{pu}}^{\prime}(t)=\frac{4 M_{1}}{\pi}\left[\sin \left(\omega_{\mathrm{s}} t+\varphi_{13}\right)+\frac{1}{3} \sin \left(3 \omega_{\mathrm{s}} t+3 \varphi_{13}\right)\right] \\
v_{\mathrm{T} 2, \mathrm{pu}}^{\prime}(t)=\frac{4 M_{2}}{\pi}\left[\sin \left(\omega_{\mathrm{s}} t+\varphi_{23}\right)+\frac{1}{3} \sin \left(3 \omega_{\mathrm{s}} t+3 \varphi_{23}\right)\right] \\
v_{\mathrm{T} 3, \mathrm{pu}}(t)=\frac{4}{\pi}\left[\sin \omega_{\mathrm{s}} t+\frac{1}{3} \sin \left(3 \omega_{\mathrm{s}} t\right)\right]
\end{array},\left\{\begin{array}{c}
M_{1}=\frac{V_{1}}{n_{13} V_{\mathrm{B}}} \\
M_{2}=\frac{V_{2}}{n_{23} V_{\mathrm{B}}} \\
Q=\frac{\omega_{r} L_{\mathrm{P} 1}}{Z_{\mathrm{B}}}
\end{array}\right.\right.
$$

Based on the assumptions above, for the transformer, the voltages of the three ports are clamped. There is a decoupling relationship between the BAT port and SC port, whose currents are only determined by the voltage of BUS port and phase shift angles respectively. Based on Equations (5) and (7), the three ports currents of transformer are approximately derived as follows:

$$
\left\{\begin{array}{l}
i_{\mathrm{T} 1, \mathrm{pu}}^{\prime}(t)=\frac{4 n_{13}^{2}}{\pi}\left[\begin{array}{c}
\frac{\cos \left(\omega_{\mathrm{s}} t\right)-M_{1} \cos \left(\omega_{\mathrm{s}} t+\varphi_{13}\right)}{X_{\mathrm{s} 1, \mathrm{pu}}}+ \\
\frac{\cos \left(3 \omega_{\mathrm{s}} t\right)-M_{1} \cos \left(3 \omega_{\mathrm{s}} t+3 \varphi_{13}\right)}{3 X_{\mathrm{s} 3, \mathrm{pu}}}
\end{array}\right] \\
i_{\mathrm{T} 2, \mathrm{pu}}^{\prime}(t)=\frac{4 n_{23}^{2}}{\pi}\left[\begin{array}{c}
\frac{\cos \left(\omega_{\mathrm{s}} t\right)-M_{2} \cos \left(\omega_{\mathrm{s}} t+\varphi_{23}\right)}{X_{\mathrm{s} 1, \mathrm{pu}}}+ \\
\frac{\cos \left(3 \omega_{\mathrm{s}} t\right)-M_{2} \cos \left(3 \omega_{\mathrm{s}} t+3 \varphi_{23}\right)}{3 X_{\mathrm{s} 3, \mathrm{pu}}}
\end{array}\right] \\
i_{\mathrm{T} 3, \mathrm{pu}}(t)=i_{\mathrm{T} 1, \mathrm{pu}}^{\prime}(t)+i_{\mathrm{T} 2, \mathrm{pu}}^{\prime}(t)
\end{array}\right.
$$

The turn-off loss of MOSFET and the condition of ZVS are directly related to the turn-off current, and thus it is necessary to make a careful analysis of the switching off current on these ports. For the full-bridge of BAT (port 1), the turn-off time of switch S2 and S3 can be given from $w_{\text {st }}=-\varphi_{13}$, since the phase shift angle of BAT is set to $\varphi_{13}$. At the same time, the turn-off time of S1 and $\mathrm{S} 4$ is $w_{\text {st }}=-\varphi_{13}+\pi$. Besides, the turn-off current of S1-S4 have the same value, because the resonant current is composed by a series of symmetrical sinusoidal components. Similarly, the turn-off time can also be obtained for the side of BUS and SC. As a result, the turn-off current of MOSFETs in three ports can be expressed as:

$$
\left\{\begin{array}{l}
i_{\mathrm{T} 1, \mathrm{pu}}^{\prime}\left(\frac{-\varphi_{13}}{\omega_{\mathrm{S}}}\right)=\frac{4 n_{13}^{2}}{\pi}\left[\frac{\cos \left(\varphi_{13}\right)-M_{1}}{X_{\mathrm{s} 1, \mathrm{pu}}}+\frac{\cos \left(3 \varphi_{13}\right)-M_{1}}{3 X_{\mathrm{s} 3, \mathrm{pu}}}\right] \\
i_{\mathrm{T} 2, \mathrm{pu}}^{\prime}\left(\frac{-\varphi_{23}}{\omega_{\mathrm{S}}}\right)=\frac{4 n_{23}^{2}}{\pi}\left[\frac{\cos \left(\varphi_{23}\right)-M_{2}}{X_{\mathrm{s} 1, \mathrm{pu}}}+\frac{\cos \left(3 \varphi_{23}\right)-M_{2}}{3 X_{\mathrm{s} 3, \mathrm{pu}}}\right] \\
i_{\mathrm{T} 3, \mathrm{pu}}(0)=\left\{\begin{array}{c}
\frac{4 n_{13}^{2}}{\pi}\left[\frac{1-M_{1} \cos \left(\varphi_{13}\right)}{X_{\mathrm{s} 1, \mathrm{pu}}}+\frac{1-M_{1} \cos \left(3 \varphi_{13}\right)}{3 X_{\mathrm{s} 3, \mathrm{pu}}}\right]+ \\
\frac{4 n_{23}^{2}}{\pi}\left[\frac{1-M_{2} \cos \left(\varphi_{23}\right)}{X_{\mathrm{s} 1, \mathrm{pu}}}+\frac{1-M_{2} \cos \left(3 \varphi_{23}\right)}{3 X_{\mathrm{s} 3, \mathrm{pu}}}\right]
\end{array}\right\}
\end{array}\right.
$$

where $-\varphi_{23}$ is the phase shift angle for SC. On the other hand, without concerning 3rd harmonic component, the cut-off currents of three ports in SR-TBC can also be derived as Equation (10):

$$
\left\{\begin{array}{l}
i_{T 1, p u}^{\prime}\left(-\frac{\varphi_{1}}{t}\right)=\frac{4 n_{13}^{2}}{\pi X_{p u}}\left(\cos \varphi_{1}-M_{1}\right) \\
i_{T 2, p u}^{\prime}\left(-\frac{\varphi_{2}}{t}\right)=\frac{4 n_{23}^{2}}{\pi X_{p u}}\left(\cos \varphi_{2}-M_{2}\right) \\
i_{T 3, p u}(0)=\frac{4 n_{13}^{2}}{\pi X_{\mathrm{pu}}}\left(1-M_{1} \cos \varphi_{1}\right)+\frac{4 n_{23}^{2}}{\pi X_{\mathrm{pu}}}\left(1-M_{2} \cos \varphi_{2}\right)
\end{array}\right.
$$

\section{Power Loss Modeling}

Loss analysis plays an important role in improving the overall performance of the converter system. In this section, the loss calculation models are established for MR-TBC and SR-TBC. Moreover, the corresponding theoretical formula will be deduced in details, which will lay a theoretical foundation for the later analysis. Before the analysis, several assumptions are made to simplify the procedure: 
(1) The effect of transformer leakage inductance and some other parasitic parameters on loss is not considered.

(2) The devices of the same type are regard as the ideal, whose parameters only follow the datasheets. Individual differences are ignored.

(3) The influence of temperature on some device parameters is ignored. If the parameter is temperature dependent, the calibration is only based on the datasheet. The analysis is carried out considering the operating temperature of the converter is $75^{\circ} \mathrm{C}$.

\subsection{MOSFET Power Loss Model}

As the main part of the loss of the power electronic converter, the calculation of the switching loss is relatively complicated. Switching loss can be divided into conduction loss $P_{\mathrm{sw} \_c o n}$, reverse conduction loss $P_{\mathrm{sw} \_\mathrm{SD}}$, output capacitor loss $P_{\mathrm{sw} \_o s s}$, turn-off $P_{\mathrm{sw} \_ \text {off }}$, turn-on loss $P_{\mathrm{sw} \_ \text {on }}$ and drive

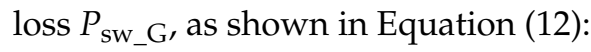

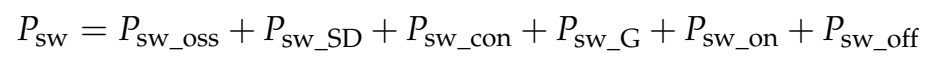

The voltage and current waveforms of switch $S_{1}$ and $S_{2}$ during the turn-on transition are shown in Figure 6. In the picture, $v_{\mathrm{S} 1}$ is the drain-source voltage of $\mathrm{S} 1 ; v_{\mathrm{G}_{-} \mathrm{S} 1}$ and $v_{\mathrm{G}_{-} \mathrm{S} 2}$ represent the gate voltages of $\mathrm{S} 1$ and $\mathrm{S} 2$, respectively; $V_{\mathrm{pl}}$ means the Miller platform voltage of the the switch. $i_{\mathrm{S} 1}$ and $i_{\mathrm{S} 2}$ are defined as the current of $\mathrm{S} 1$ and $\mathrm{S} 2 ; t_{\mathrm{db}}$ is the dead band time; $t_{\mathrm{r}}$ represents the gate voltage rise time of switch; $t_{\mathrm{oss}}$ is defined as the output capacitance discharge time of the switch; $t_{\mathrm{SD}}$ means the time of reverse conduction.

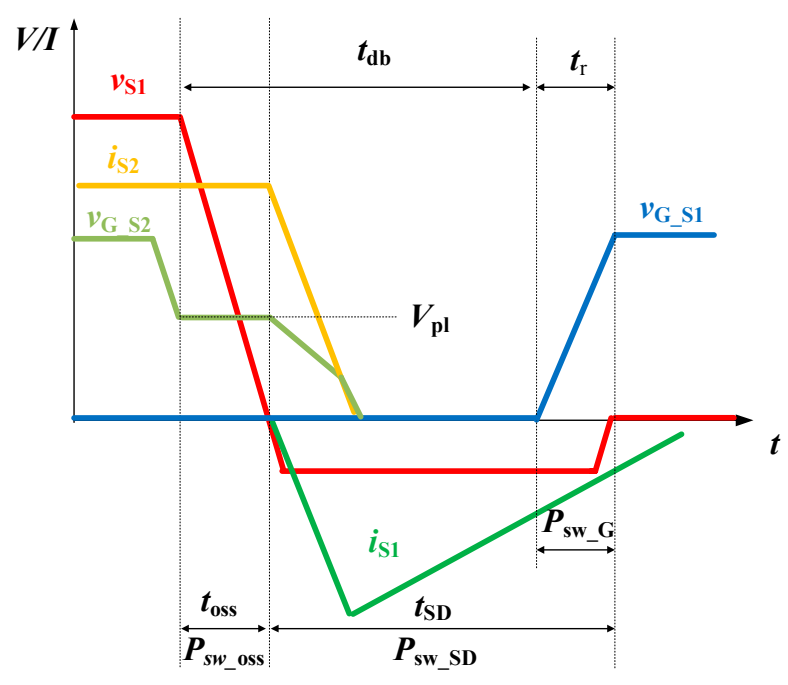

Figure 6. Current and voltage waveforms during the turn-on transition.

When $\mathrm{S}_{2}$ is turned off, after a short delay until $v_{\mathrm{G} \_\mathrm{S} 2}$ drops to $V_{\mathrm{pl}}$, the output capacitor $C_{\mathrm{oss}}$ of $\mathrm{S} 1$ begins to discharge while $C_{\mathrm{oss}}$ of $\mathrm{S} 2$ starts to charge. At the same time, $v_{\mathrm{S} 1}$ begins to decline, while $v_{\mathrm{S} 2}$ rises. In order to achieve ZVS, $v_{S 1}$ must be reduced to 0 before $v_{\mathrm{G}_{-} S 1}$ reaching a high level. According to [31], $t_{\mathrm{oss}}$ can be given as Equation (13), where $I_{\text {off }}$ represents turn-off current; $Q_{\mathrm{S} 1}$ and $Q_{\mathrm{S} 2}$ are the output charge of $S_{1}$ and $S_{2}$ :

$$
t_{\mathrm{oss}}=\frac{Q_{\mathrm{S} 1}+Q_{\mathrm{S} 2}}{I_{\mathrm{off}}}
$$


When $t_{\mathrm{oss}}<t_{\mathrm{db}}$, the turn-on switching losses $P_{\mathrm{sw} \_ \text {on }}$ and $C_{\mathrm{oss}}$ discharging losses $P_{\text {sw_oss }}$ are eliminated in ZVS conditions. However, when $t_{\mathrm{oss}}>t_{\mathrm{db}}, P_{\mathrm{sw} \_}$on and $P_{\mathrm{sw} \_ \text {oss }}$ are unavoidable, which can be calculated as Equations (13) and (14):

$$
\begin{gathered}
P_{\mathrm{sw} \_ \text {oss }}=f_{\mathrm{sw}} \cdot \int_{0}^{V_{\mathrm{in}}} v_{\mathrm{DS}} C_{\mathrm{oss}}\left(v_{\mathrm{DS}}\right) \mathrm{d} v_{\mathrm{DS}} \\
P_{\mathrm{sw} \_ \text {on }}=f_{\mathrm{sw}} \cdot \int_{0}^{t_{\mathrm{x}}} v_{\mathrm{DS}}(t) i_{\mathrm{DS}}(t) \mathrm{d} t
\end{gathered}
$$

where $i_{\mathrm{DS}}$ represents the current of switch in turn-on transition; $t_{\mathrm{x}}$ means the overlap time between $v_{\mathrm{DS}}$ and $i_{\mathrm{DS}} ; C_{\mathrm{oss}}$ means the output capacitance value of the switch, which is affected by the drain-source voltage $v_{\text {DS }}$ and exhibits nonlinear characteristics. Therefore the method of piecewise integral is adopted to deal with this problem in this paper. The function curve is given as Figure 7.

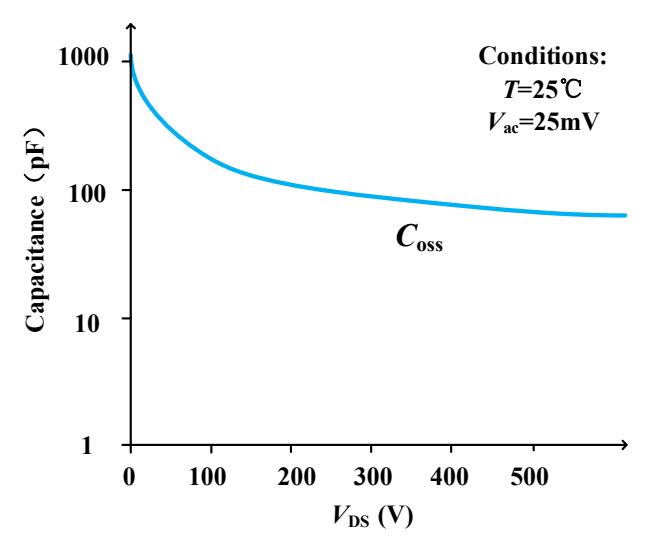

Figure 7. Curves of $C_{\mathrm{oss}}$ and $V_{\mathrm{DS}}$.

When $v_{S 1}$ drops to $0, I_{S 1}$ begins flow through parallel diode of $S 1$ until $v_{G_{-} S 1}$ reaches a high level. In this stage, as the current through the diode will produce a reverse voltage resulting in $P_{\text {Sw_SD }}$ can be defined by Equation (15). Where $V_{\mathrm{SD}}$ can be checked from the datasheet of switch, and the time of reverse conduction $t_{\mathrm{SD}}$ can be calculated by $t_{\mathrm{oss}}, t_{\mathrm{db}}$ and $t_{\mathrm{r}}$ :

$$
P_{\mathrm{SW} \_\mathrm{SD}}=f_{\mathrm{SW}} \cdot \int_{0}^{t_{\mathrm{SD}}} V_{\mathrm{SD}}(t) i_{\mathrm{SD}}(t) \mathrm{d} t_{\mathrm{SD}}
$$

In this process, in order to achieve the ZVS, it is necessary to ensure that $\mathrm{S} 1$ is turned on before the change of current $i_{\mathrm{s} 1}$ direction. As Figure 6 shows, the Miller platform does not exist during the turn-on transition of $\mathrm{S} 1$ under the $\mathrm{ZVS}$ condition. $P_{\mathrm{SW}_{-} \mathrm{G}}$ switch drive loss $P_{\mathrm{Sw}_{-} \mathrm{G}}$ can be expressed as (16) under the ZVS condition, where, $Q_{\mathrm{G}}$ is the charge required for gate conduction, and $Q_{\mathrm{GD}}$ is the charge required for Miller platform, but in the paper, an external source is used as the driving power for experiments, and thus the $P_{\mathrm{SW}_{-} \mathrm{G}}$ is not considered in the calculation of efficiency and loss:

$$
P_{\mathrm{sW}_{-} \mathrm{G}}=f_{\mathrm{sW}} V_{\mathrm{GS}}\left(Q_{\mathrm{G}}-Q_{\mathrm{GD}}\right)
$$

At the stage, when $i_{\mathrm{s} 1}$ flows through $\mathrm{S} 1$, the $P_{\mathrm{sw} \_ \text {con }}$ is caused by the conduction resistance $R_{\mathrm{ds}}$ of S1 shown as:

$$
P_{\mathrm{sw} \_ \text {con }}=I_{\mathrm{S} 1}^{2} R_{\mathrm{ds}}
$$

Although the converter can eliminate the $P_{\mathrm{Sw} \_o n}$ by ZVS, $P_{\mathrm{Sw} \_ \text {off }}$ cannot be avoided. In order to better analyze $P_{\mathrm{Sw} \_ \text {off }}$, the voltage and current waveforms during the turn-off transition are described as Figure 8 , where $v_{\mathrm{S} 2}$ means the source-drain voltage of $\mathrm{S} 2, V_{\text {th }}$ is the gate threshold voltage of the switch. According to [31], $P_{\text {sw_off }}$ can be divided into two parts. In $t_{\text {off1 }}, i_{S 2}$ keeps constant, while 
$v_{\mathrm{S} 2}$ increases linearly; in $t_{\mathrm{off} 2}, v_{\mathrm{S} 2}$ keeps constant, while $i_{\mathrm{S} 2}$ decreasing linearly. As a result, $P_{\mathrm{SW} \_ \text {off }}$ can be expressed as:

$$
\begin{gathered}
P_{\mathrm{off}}=\frac{f_{\mathrm{sw}} I_{\mathrm{off}} V_{\mathrm{in}}}{2}\left(t_{\mathrm{off} 1}+t_{\mathrm{off} 2}\right) \\
t_{\mathrm{off} 1}=\frac{Q_{\mathrm{GD}} R_{\mathrm{Goff}}}{V_{\mathrm{pl}}}, t_{\mathrm{off} 2}=\frac{Q_{\mathrm{GS}}\left(R_{\mathrm{Goff}}+R_{\mathrm{s} 1}\right)}{\left(V_{\mathrm{pl}}+V_{\mathrm{th}}\right) / 2}
\end{gathered}
$$

where $R_{\mathrm{G}}$ is gate equivalent impedance; $Q_{\mathrm{GS}}$ represents the storage charge between the gate and source; $R_{\mathrm{sl}}$ is the source equivalent series impedance.

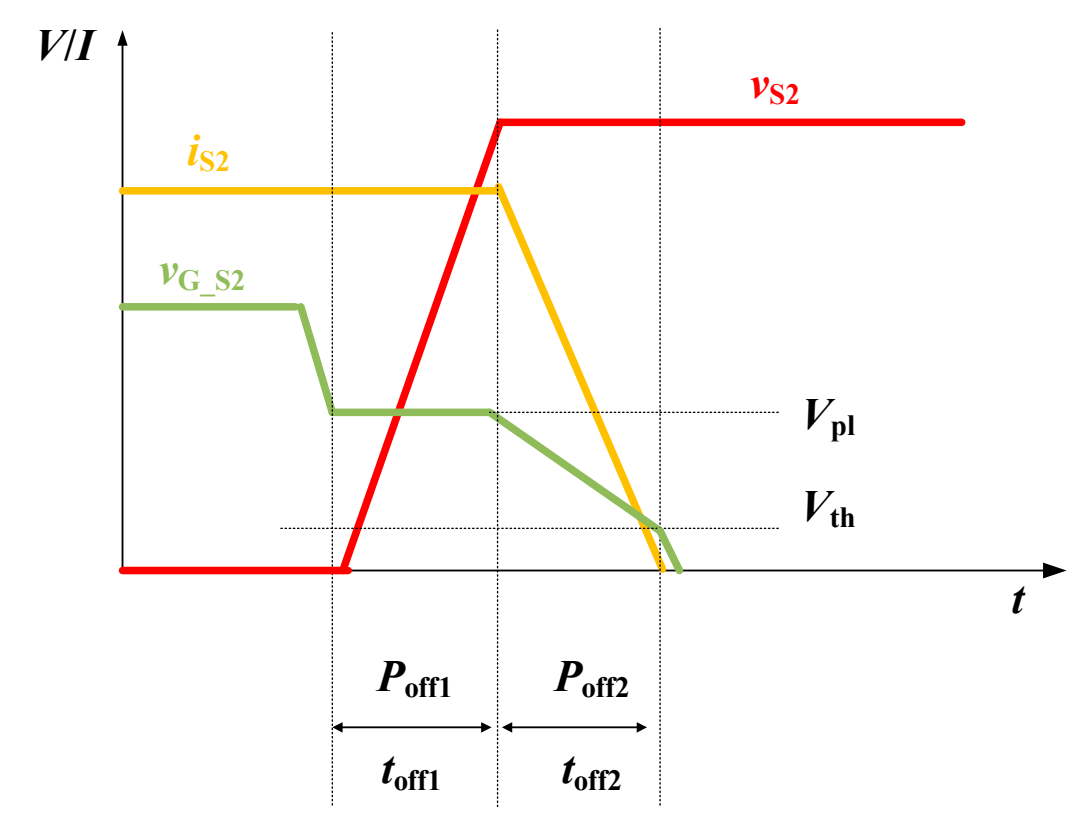

Figure 8. Current and voltage waveforms during the turn-off transition.

\subsection{Transformer Power Loss Model}

The transformer design is also a significant factor to achieve high efficiency and reduce loss for MR-TBC and SR-TBC. The transformer loss is generally divided into the core loss and copper loss.

The core loss mainly consists of the hysteresis loss and eddy current loss. The hysteresis phenomenon occurs resulting in loss when the AC current flows through the transformer. The hysteresis loss is proportional to the area enclosed by the hysteresis loop. On the other hand, when alternating magnetic lines of flux pass through the core, an induced electromotive force is generated, resulting in an electric current loop causing eddy current losses. At present, for the transformer core loss $P_{\text {core_tra, }}$ the Steinmetz equation is used to estimate, written as:

$$
P_{\text {core_tra }}=k V_{\text {eq_tra }} f_{\text {sw }}^{\alpha} B_{\max }^{\beta}
$$

where $V_{\text {eq_tra }}$ represents the equivalent volume of the transformer core; $k$ is the material factor, $\alpha$ and $\beta$ are the Steinmetz coefficients, related to the material of core. $B_{\max }$ is the peak flux density, which is influenced by the maximum excitation current and is given as:

$$
B_{\max }=\frac{I_{\max } L_{\mathrm{m}}}{2 n_{\mathrm{lp}} A_{\text {eq_tra }}}
$$

where $I_{\max }$ is the peak current through the transformer, $L_{\mathrm{m}}$ is the excitation inductance of the transformer, $n_{\mathrm{lp}}$ is the turn number of coils, and the $A_{\text {eq_tra }}$ is the equivalent cross sectional area of the transformer core. 
The copper loss is produced by the windings, mainly resulting from AC equivalent resistance of coil and current. The AC equivalent resistance $R_{\mathrm{ac}}$ will be much larger than the DC resistance of $R_{\mathrm{dc}}$ due to the proximity effect and skin effect. The skin effect causes the current to distribute unevenly in the conductor. The proximity effect causes current to flow near the conductor. The two phenomena both reduce the effective flow area of the current and increase the equivalent impedance of the winding. According to [32,33], the AC-DC resistance ratio FR at $n^{\text {th }}$ harmonic frequency can be calculated by the Dowell equation, as shown in Equation (22):

$$
F R(n, p, x)=x \cdot \frac{e^{2 x}-e^{-2 x}+2 \sin (2 x)}{e^{2 x}+e^{-2 x}+2 \cos (2 x)}+2 x \cdot \frac{p^{2}-1}{3} \cdot \frac{e^{x}-e^{-x}+2 \sin (x)}{e^{x}+e^{-x}+2 \cos (x)}
$$

where $p$ represents the number of layers in magnetic component winding; $x$ is the intermediate variable as (23), when foil is used as the coil. $h_{\text {foil }}$ is the thickness of the foil; $\delta(n)$ represents the skin depth of $n$th harmonics frequency, which can be shown as (24):

$$
\begin{gathered}
x=\frac{h_{\mathrm{foil}}}{2 \delta(n)} \\
\delta(n)=\sqrt{\frac{2 \rho_{\mathrm{Cu}}}{2 \pi n f_{\mathrm{sw}} \mu_{0}}}
\end{gathered}
$$

where $\rho_{\mathrm{Cu}}$ refers to the resistivity of copper, $\mu_{0}$ is the permeability of vacuum. In order to make the calculation of the copper loss more accurate, the Fast Fourier Transform (FFT) is employed for the current so that the copper loss at each harmonic frequency can be calculated. Thus, the transformer copper loss can be obtained by summing the losses from the 1st to 28th harmonics. Taking the BUS side as an example, the corresponding copper loss $P_{\text {cu_tra_bus }}$ can be expressed as:

$$
P_{\text {cu_tra_bus }}=R_{\text {tra_bus }} \sum_{n=1}^{28} F R_{\text {bus }} I_{\text {n_bus }}^{2}
$$

where $R_{\text {tra_bus }}$ is the winding DC resistance of BUS side. The copper loss of the other ports $\left(P_{\text {cu_tra_bat }}\right.$ and $\left.P_{\text {cu_tra_sc }}\right)$ can be calculated by the same method. Then, the total copper loss of transformer $P_{\text {cu_tra }}$ can be deduced as:

$$
P_{\text {cu_tra_bus }}=P_{\text {cu_tra_bus }}+P_{\text {cu_tra_bat }}+P_{\text {cu_tra_sc }}
$$

\subsection{Resonant Inductor Power Loss Model}

The resonant inductors is applied in the SR-TBC or MR-TBC, as shown in Figure 2. Similar to the transformer, the losses in the resonant inductors also consist of copper loss and core loss. Thereby, the loss of inductors can be derived by the same method used in the transforms, as follows:

$$
\begin{gathered}
P_{\text {core } \_\mathrm{L}}=k V_{\text {eq_L }} f_{\mathrm{sw}}^{\alpha} B_{\mathrm{L} \_ \text {max }}^{\beta}, B_{\mathrm{L} \_ \text {max }}=\frac{I_{\mathrm{L} \_\max } L}{2 n_{\mathrm{lp} \_\mathrm{L}} A_{\text {eq_L }}} \\
P_{\text {cu_L } \_ \text {bus }}=R_{\mathrm{L}} \sum_{n=1}^{28} F_{\mathrm{R} \_\mathrm{L}} I_{\mathrm{n} \_\mathrm{L}}^{2}
\end{gathered}
$$

where $R_{\mathrm{L}}$ is the equivalent resistance of the resonant inductance. However, the resonant inductor used a multi strand Liz wires as the excitation coil instead of copper foil. Therefore, the Formula (23) used for foils is no longer applicable. The expression for the intermediate variable $x$ of $F_{R_{-} L}$ is rewritten as (28), where, $d_{\mathrm{awg}}$ is the diameter of the conductor cross-section:

$$
x=\frac{\sqrt{\pi} d_{\mathrm{awg}}}{2 \delta(n)}
$$




\subsection{Capacitors Power Loss Model}

The resonant capacitors and filter capacitors are used in the MR-TBC and SR-TBC. The losses of capacitors also takes up a certain share of the total loss, which cannot be ignored. The resonant capacitor needs to withstand high current and high voltage. To achieve high efficiency and a low temperature rise, the MKP metallized polypropylene film capacitor is selected owing to a smaller dissipation factor $(\tan \delta)$. The equivalent series resistance (ESR) of each resonant capacitor $R_{\mathrm{Cr}}$ can be calculated according to $\tan \delta$ and the capacitance, given as:

$$
R_{\mathrm{Cr}}=\frac{\tan (\delta)}{2 \pi f_{\mathrm{sW}} C_{\mathrm{R}}}
$$

The loss of resonant capacitance $P_{\mathrm{Cr}}$ is resulting from the RMS of resonant current $I_{\mathrm{cr}}$ and $R_{\mathrm{Cr}}$, which can be deduced as:

$$
P_{\mathrm{Cr}}=I_{\mathrm{Cr}}^{2} R_{\mathrm{Cr}}
$$

In the converter, filter capacitors are generally used for DC voltage regulation of input and output. Their losses are calculated according to the flowing ripple current $I_{\text {rip }}$ and the equivalent series resistance of each filter capacitor $R_{C}$, wherein the ripple current has been calculated by summing the frequency harmonic components (DC component excluded) from the 1st to the 28th frequency. In this way, the three-port ripple current $I_{\text {rip_bat }}, I_{\text {rip_sc }}$ and $I_{\text {rip_bus }}$ can be obtained and thus the three-port filter capacitor loss $P_{\mathrm{c} \_ \text {bat }}, P_{\mathrm{c} \_ \text {sc }}$ and $P_{\mathrm{c} \_ \text {bus }}$ can be approximately expressed as follows:

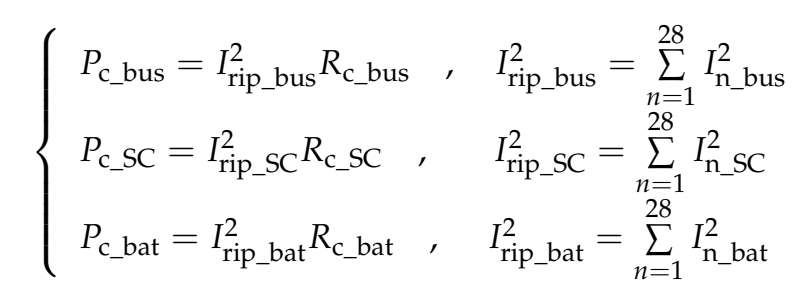

In order to ensure the consistency of the experiments, the same filter capacitor are used in experiments of the MR-TBC and SR-TBC. Besides, the MKP metallized polypropylene film capacitors are connected in parallel here, which can further reduce $R_{\mathrm{c}}$ and avoid excessive power loss.

\section{Loss Distribution Analysis and Optimization}

\subsection{Loss Distribution Comparison between Si and SiC MOSFET}

Based on the calculation methods mentioned above, the loss distribution of MR-TBC with a Si MOSFET is shown in Figure 9a while the corresponding loss distribution of a SiC MOSFET is shown in Figure 9b. The IPW65R041CFD is adopted for the Si MOSFET, which is described as the most suitable for phase shift control. From the figure, it can be found that the switch losses take the major part of the total loss in both cases (over $60 \%$ at rating work condition). For the switch losses, the $P_{\text {sw_on }}$ and $P_{\text {sw_oss }}$ can be overlooked due to the ZVS characteristics. At the same time, $P_{\text {Sw_sd }}$ and $P_{\text {sw_G }}$ are also limited to a very small range due to the phase shift control strategy, while, $P_{\text {sw_con }}$ and $P_{\text {sw_off }}$ became the principal reason for inefficiency. The Si MOSFET has a slightly better performance than $\mathrm{SiC}$ in $P_{\mathrm{sw}}$ con since the parameter $R_{\mathrm{ds}}$ of the Si MOSFET used is smaller than for SiC. However, in the part of $P_{\text {sw_off }}$ Si devices caused more losses than SiC due to the parameter $Q_{G}$ and $Q_{G D}$. More seriously, an additional loss, the turn-off loss of body diode of the MOSFET $P_{\text {doff }}$ is introduced during the turn-off transition, which accounts for $10 \%$ of the total loss. Fortunately, the SiC switch overcomes this shortcoming of Si devices. Due to the inherent nature of $\mathrm{SiC}$ devices, the reverse recovery time is consider to be 0 , and $P_{\text {doff }}$ can be eliminated and the $P_{\text {sw }}$ is limited to less than $60 \%$. 


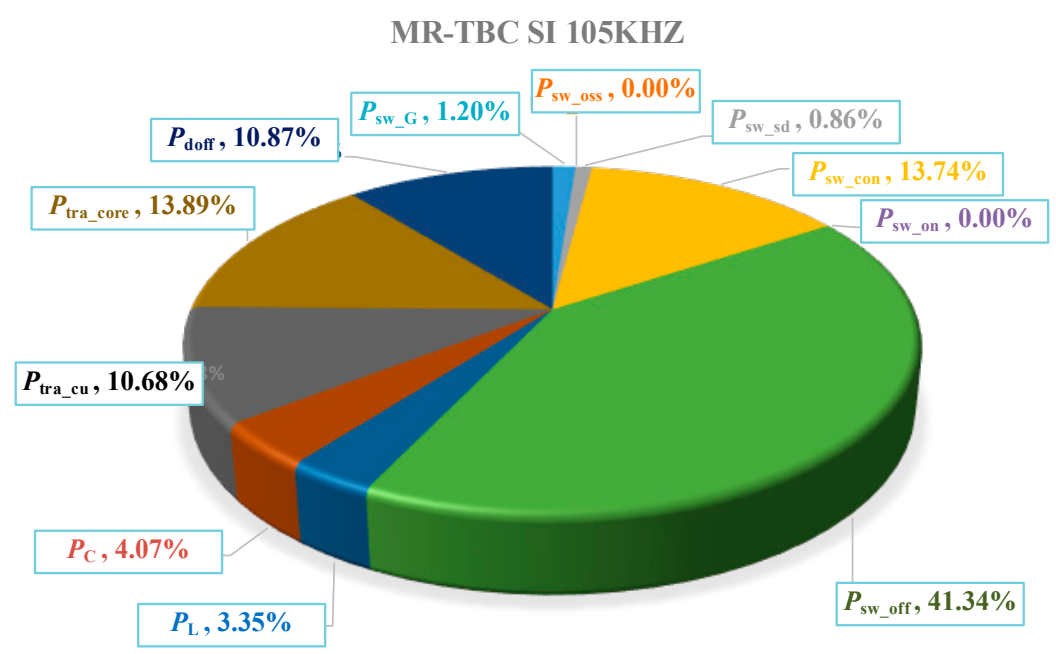

(a)

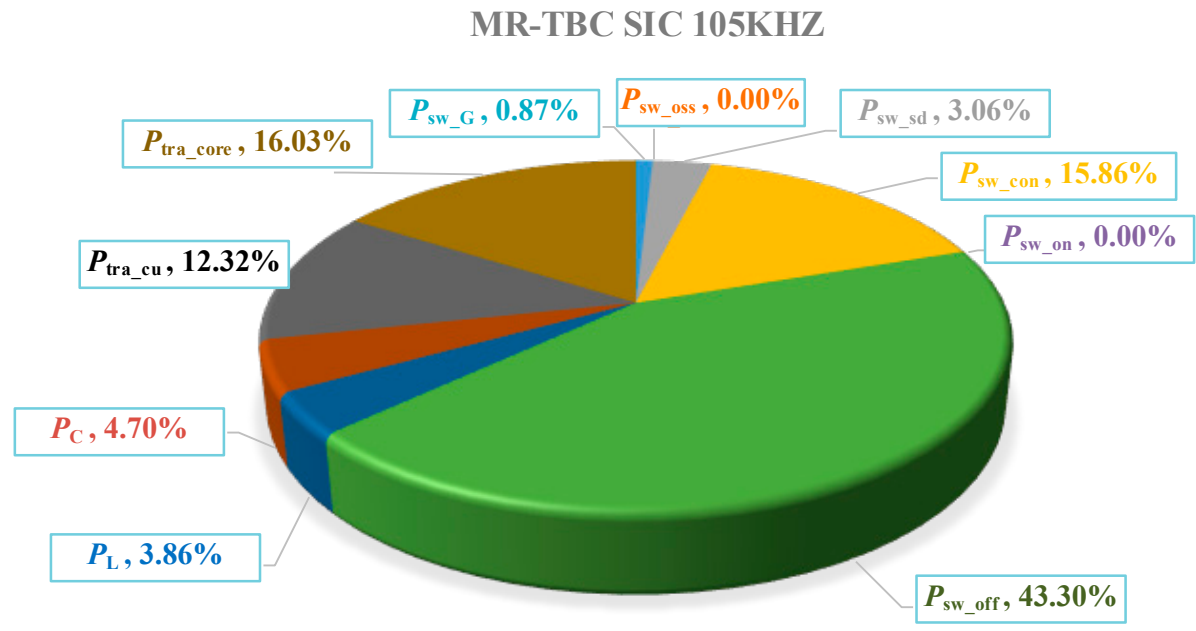

(b)

Figure 9. Loss distribution comparison between Si and SiC under $1500 \mathrm{~W}$ (a) Si; (b) SiC.

The comparative calculation example is set up to demonstrate the advantages of the SiC switch in efficiency, as shown in Figure 10. Compared to the Si device, the SiC MOSFET cause more losses in $P_{\mathrm{sw} \_ \text {con, }}$ since $R_{\mathrm{ds}}$ is larger than the Si device $\left(70 \mathrm{~m} \Omega\right.$ for $\mathrm{SiC}$ and $55 \mathrm{~m} \Omega$ for $\mathrm{Si}$ at $\left.75^{\circ} \mathrm{C}\right)$. However, the $\mathrm{SiC}$ MOSFET makes a greater contribution in restraining $P_{\text {doff. }} . P_{\mathrm{sw} \_ \text {off }}$ is also relieved to a certain extent due to the lower $Q_{\mathrm{G}}$ and $Q_{\mathrm{GD}}$. Besides, $P_{\mathrm{Sw} \_\mathrm{G}}$ and $P_{\mathrm{Sw} \_}$sd are also ameliorated, but the effect is not obvious. This is because $P_{\text {SW_G }}$ and $P_{\text {SW_sd }}$ represent a very limited share of total losses for MR-TBC.

As a result, the $\mathrm{SiC}$ MOSFET shows better characteristics and its losses account for only $87 \%$ of the losses produced by using the Si devices. The converter harvests a better efficiency by using SiC devices. Therefore, the SiC MOSFET is selected in the following analysis. 


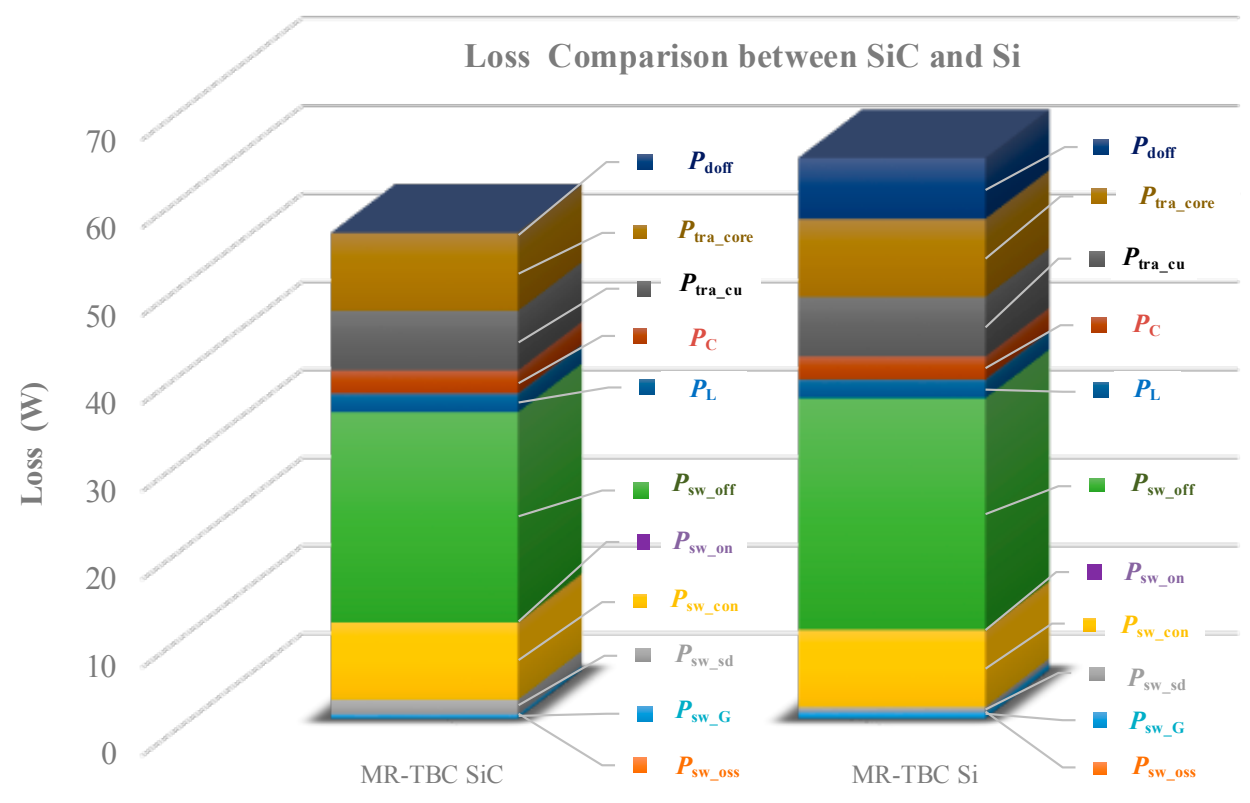

Figure 10. Loss calculation comparison between Si and SiC under $1500 \mathrm{~W}$.

\subsection{Loss Comparison between $S R-T B C$ and $M R-T B C$}

Based on the above analysis, theoretical calculations are carried out for MR-TBC and SR-TBC in the conditions of 1500, 1000 and $500 \mathrm{~W}$. In this way, the operating characteristics and efficiency curves of the converters under full load conditions can be obtained. Taking the forward operation as an example, the loss distributions of two topologies under a full load range are analyzed and compared. From the results of the corresponding calculations, the strengths and weaknesses of the two topologies will be harvested from the two aspects of efficiency and ZVS range.

In the loss calculations, the resonant component parameters of the MR-TBC and SR-TBC are both designed to achieve ZVS under rated power $1500 \mathrm{~W}$ and working frequency $105 \mathrm{kHz}$. The detailed parameters are summarized in Table 1 . The dead-band time $t_{\mathrm{db}}$ is set to $150 \mathrm{~ns}$.

Table 1. Parameters of the proposed MR-TBC and SR-TBC.

\begin{tabular}{|c|c|c|c|}
\hline \multicolumn{2}{|c|}{ Element } & MR-TBC & SR-TBC \\
\hline \multicolumn{2}{|c|}{ Switch } & \multicolumn{2}{|c|}{ SiC MOSFET Type: C3M0065090D } \\
\hline \multicolumn{2}{|c|}{ Transformer } & \multicolumn{2}{|c|}{$\begin{array}{l}\text { Core EE } 85 * 2 \text { in parallel } \\
\text { turns ratio } n_{\mathrm{BUS}}: n_{\mathrm{BAT}}: n_{\mathrm{SC}}=14: 5.25: 7\end{array}$} \\
\hline \multirow{4}{*}{ Resonant inductor } & Core & PQ4040 & PQ5050 \\
\hline & Turn numbers & 11.5 & 18 \\
\hline & & BAT: $L_{\mathrm{R}} 34 \mathrm{u} L_{\mathrm{P}} 31.88 \mathrm{u}$ & BAT: $L_{\mathrm{R}} 127.58 \mathrm{u}$ \\
\hline & Inductance & SC: $L_{\mathrm{R}} 33.76 \mathrm{u} L_{\mathrm{P}} 31.85 \mathrm{u}$ & SC: $L_{\mathrm{R}} 127.63 \mathrm{u}$ \\
\hline \multicolumn{2}{|c|}{ Resonant capacitance } & $\begin{array}{l}\text { Cr: FKP 37n } \\
\text { Cp: FKP 22n }\end{array}$ & Cr: FKP 22n \\
\hline \multicolumn{2}{|c|}{ Filter capacitor } & \multicolumn{2}{|c|}{$\begin{array}{l}\text { BUS: MKP } 30 u * 3 \text { and } 20 u * 2 \text { in parallel } \\
\text { SC: MKP } 30 u * 3 \text { in parallel } \\
\text { BAT: PAR } 20 u * 3 \text { in parallel }\end{array}$} \\
\hline
\end{tabular}

(1) 1500 W Output Condition

The phase-shift control strategy is adopted. According to the Equations (9) and (10), $I_{\text {off }}$ can be elevated and ZVS can be realized by changing the phase shift angles. The phase shift angles are set to 
$17^{\circ}$ and $20^{\circ}$ in the MR-TBC while these of the SR-TBC are set to $28^{\circ}$ and $39^{\circ}$ in SR-TBC. By this way, ZVS is acquired by the MR-TBC and SR-TBC. At the same time, the corresponding loss analysis results can be obtained respectively, as shown in Figure 11.

As the diagram indicated, $P_{\mathrm{sw}}$ and $P_{\text {tra }}$ have become the main factors affecting the efficiency. For $P_{\mathrm{sw}}$, the $P_{\mathrm{sw} \_ \text {on }}$ and $P_{\mathrm{sw} \_o s s}$ are eliminated in both topologies due to ZVS. $P_{\mathrm{Sw} \_ \text {off }}$ is still the main part of $P_{\mathrm{sw}}$, which is larger in the MR-TBC. This is because the 3rd harmonic of current is introduced by MR-TBC, resulting in an increase in the turn-off current. Fortunately, the 3rd current harmonic also brings benefits, which can reduce the peak current, contributing to lower $P_{\text {tra_core }}$ and $P_{\mathrm{L}}$ in the MR-TBC. In the other part such as $P_{\mathrm{sw} \_}$con, $P_{\mathrm{C}}, P_{\mathrm{sw}{ }_{-} \mathrm{G}}$ and so on, there are also some differences between SR-TBC and MR-TBC, but not significant.

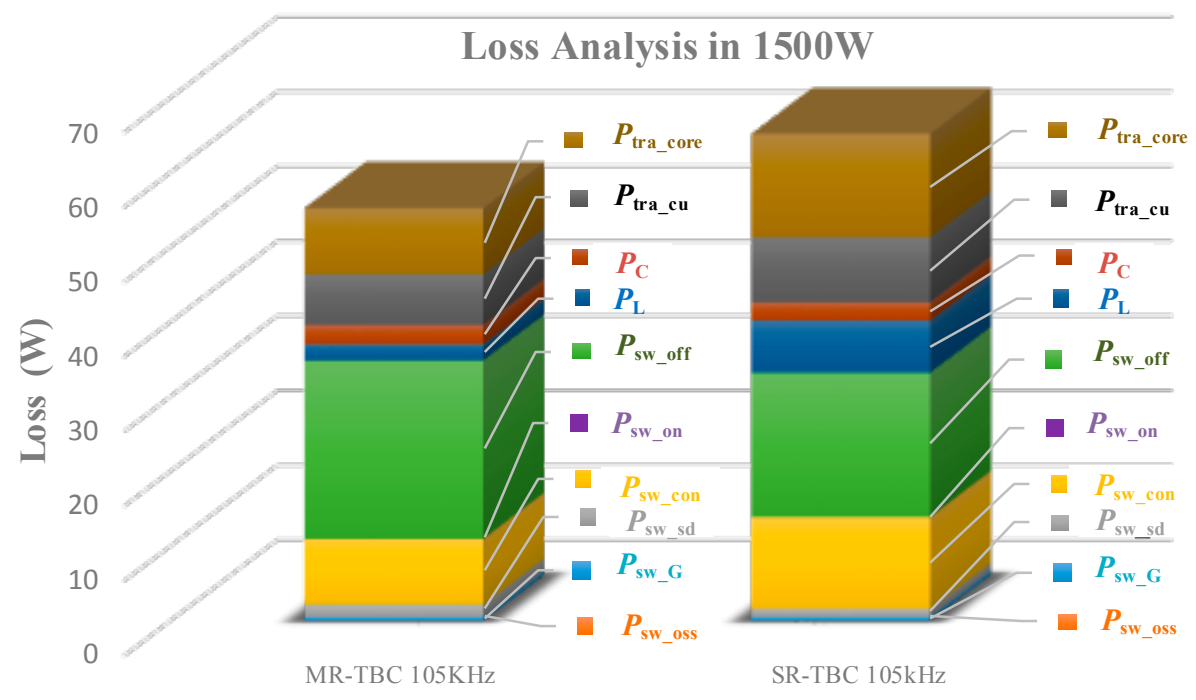

Figure 11. Loss calculation comparison of MR-TBC and SR-TBC under $1500 \mathrm{~W}$.

From the results, ZVS is realized in MR-TBC as well as SR-TBC, but the computational results show that MR-TBC has better efficiency.

\section{(2) 1000 W Output Condition}

In this case, the resonant currents decrease as the load increases, and thus the cut-off currents decline as well. Based on Equation (12), the reduction of the turn-off current will prolong the $t_{\mathrm{oss}}$. If $t_{\mathrm{oss}}>t_{\mathrm{bd}}$, ZVS cannot be acquired. On the other hand, the resonant current will decrease in $t_{\mathrm{oss}}$. If $C_{\text {oss }}$ has not yet completed the discharge before the resonant current drops to 0 , the $C_{\text {oss }}$ will be charged reversely. In this case, ZVS is also not achieved by changing the dead-band time.

According to [23], under the conditions of constant output, $I_{\text {off }}$ can be raised by increasing frequency and selecting suitable phase shift angles. By this way, ZVS can be realized when $f_{\mathrm{sw}}$ is set to $110 \mathrm{k}$ for MR-TBC while $125 \mathrm{kHz}$ for SR-TBC. In order to compare the characteristics of the two topologies, three groups of calculations are carried out: the MR-TBC working in $110 \mathrm{kHz}$, the SR-TBC working in $110 \mathrm{kHz}$ and $125 \mathrm{kHz}$. The results are shown in Figure 12.

The figure indicates that the MR-TBC realizes ZVS while SR-TBC does not when $f_{\mathrm{sw}}$ is set to $110 \mathrm{kHz}$. This is possibly attributed to the superposition of 3rd harmonic current by the MR-TBC. And $I_{\text {off }}$ can be increased so that of $C_{\text {oss }}$ can completed the discharge in $t_{\mathrm{db}}$. In contrast, for the SR-TBC, $C_{\text {oss }}$ cannot discharge fully and causes current oscillation which introduces extra losses such as $P_{\text {Sw_on }}$

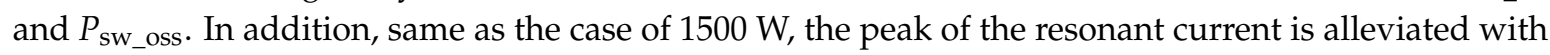
the introduction of $3 \mathrm{rd}$ harmonic current by the MR-TBC. And the good response has been achieved in $P_{\text {tra }}$ and $P_{\mathrm{L}}$ which are reduced to half of the loss generated by SR-TBC $110 \mathrm{kHz}$. As $I_{\text {off }}$ is higher, the more turn-off losses have been created than SR-TBC, but the excess losses is limited compared 
to the advantages of MR-TBC. On the other hand, for SR-TBC the ZVS can be acquired in $125 \mathrm{kHz}$, $P_{\mathrm{SW} \_ \text {oss }}$ and $P_{\mathrm{Sw} \_ \text {on }}$ can be avoided. Unfortunately, the higher working frequency and greater turn-off current cause more losses as a whole which make its efficiency suffer from progressive reduction.

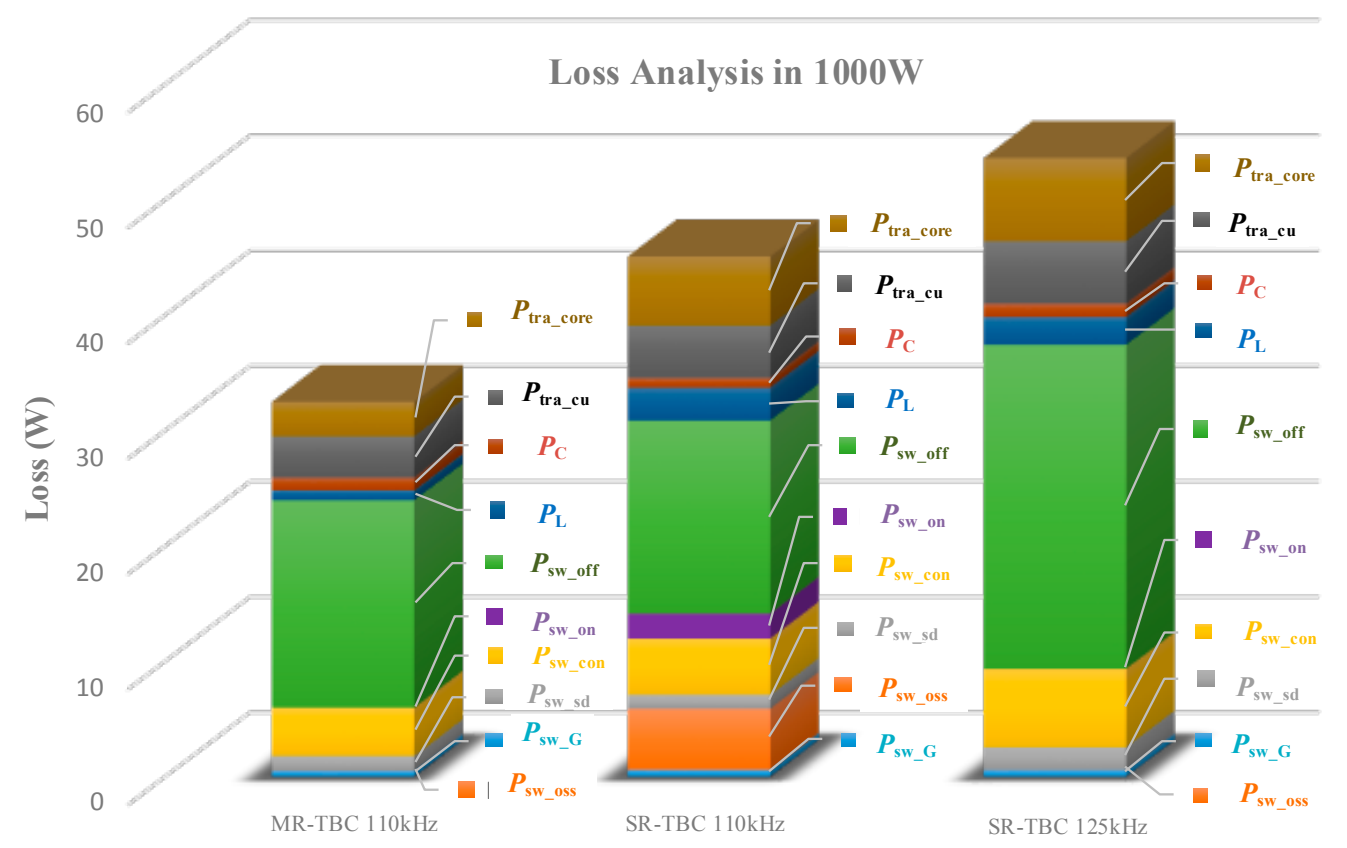

Figure 12. Loss calculation comparison of MR-TBC and SR-TBC under $1000 \mathrm{~W}$.

As a result, in the case of $1000 \mathrm{~W}$, MR-TBC harvest higher efficiency and wider ZVS range than SR-TBC due to the introduction of 3rd harmonic current.

\section{(3) 500 W Output Condition}

At $500 \mathrm{~W}$, with $I_{\text {off }}$ decreasing, and the condition of realizing ZVS becomes more severe. To obtain higher $I_{\text {off }}, f_{\text {sw }}$ need be increased further. According to the result of calculation, ZVS can be achieved by MR-TBC as $f_{\mathrm{sw}}$ is equal to $145 \mathrm{kHz}$, while SR-TBC needs more than $160 \mathrm{kHz}$. Unfortunately, efficiency would be sacrificed when $f_{\mathrm{sw}}$ is too high. On the contrary, although ZVS cannot be acquired for the MR-TBC at $f_{\mathrm{sw}}=130 \mathrm{kHz}, C_{\text {oss }}$ discharge is almost completed, which can be regarded as the quasi ZVS. By this way, the best efficiency can be harvested. So, the loss analysis is implemented in the situation of $130 \mathrm{kHz}$ and $150 \mathrm{kHz}$ by the SR-TBC and MR-TBC, as shown in Figure 13.

As depicted in the picture, $P_{\mathrm{SW} \_ \text {on }}$ exists in the three cases, but when $f_{\mathrm{sw}}$ is set to $130 \mathrm{kHz}, P_{\mathrm{sw} \_ \text {on }}$ produced by MR-TBC is much smaller than that in SR-TBC. This is because $I_{\text {off }}$ of MR-TBC is larger and the quasi ZVS is realized, resulting in limited $P_{\mathrm{SW} \_ \text {on }}$ and $P_{\mathrm{Sw} \_ \text {oss }}$. However, similar to $1000 \mathrm{~W}$ and 1500 , the introduction of $3 r d$ harmonic current by MR-TBC caused a sharp rise in turn-off loss. $P_{\text {Sw_off }}$ of MR-TBC is much larger than SR-TBC at the same working frequency. This is a challenge that need to be improved in the future work. But, in the other parts such as $P_{\operatorname{tra}}, P_{\mathrm{L}}$ and $P_{\mathrm{sw} \_ \text {oss }}, \mathrm{MR}-\mathrm{TBC}$ greatly reduces these losses to an acceptable range, compared to the SR-TBC. Therefore, the MR-TBC has shown its superiority by reducing the loss to $2 / 3$, and higher efficiency is achieved over a wider range.

As can be seen from the three cases, although the introduction of 3rd harmonic current by MR-TBC leads to some turn-off losses, MR-TBC exhibits excellent overall efficiency and ZVS range characteristics. 


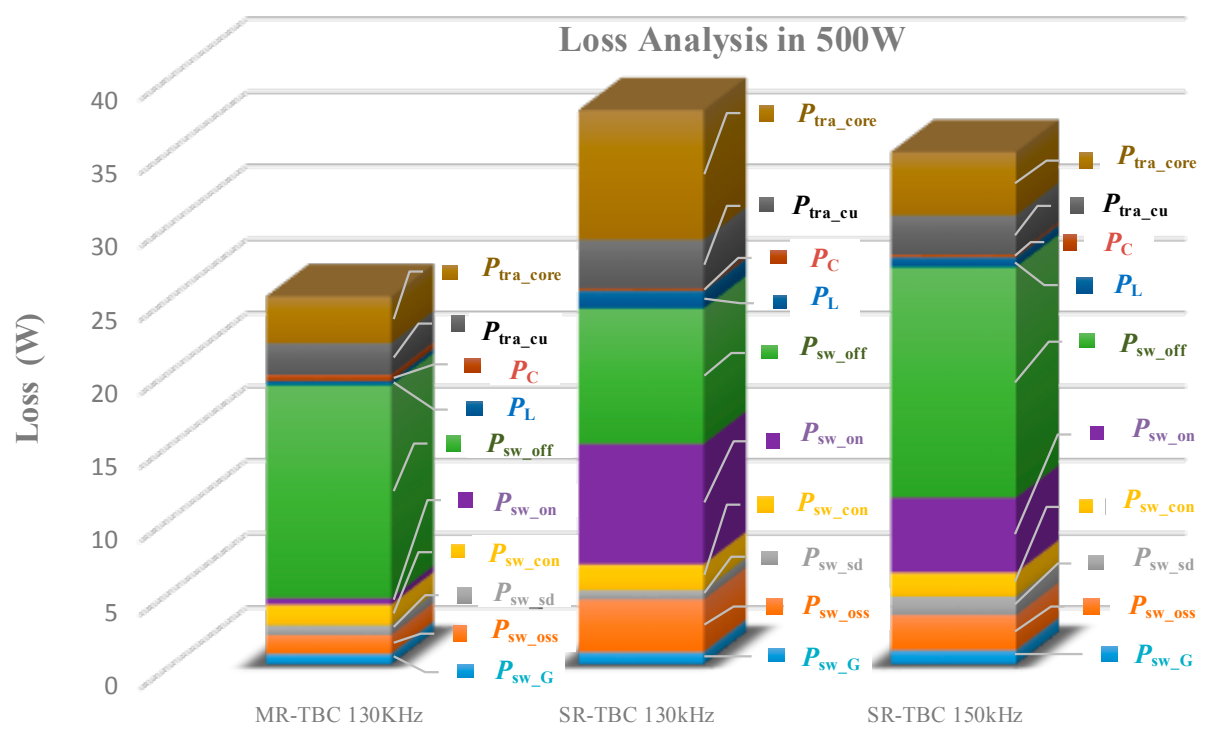

Figure 13. Loss calculation comparison of MR-TBC and SR-TBC under $500 \mathrm{~W}$.

\section{Experimental Results}

In order to verify the accuracy of theoretical analysis, prototypes of MR-TBC and SR-TBC are established. The corresponding experiments are carried out for the conditions of 1500, 1000 and $500 \mathrm{~W}$. The experimental parameters are listed in Table 1.

\section{(1) 1500 W Output Condition}

Under a rated load of $1500 \mathrm{~W}$, the switching frequency $f_{\mathrm{sw}}$ is set to $105 \mathrm{kHz}$, and thus the three ports experimental waveforms of MR-TBC and SR-TBC are shown in Figure 14. Figure 14b,d,f,h,j,l are zoom-in versions of Figure 14a,c,e,g,i,k.

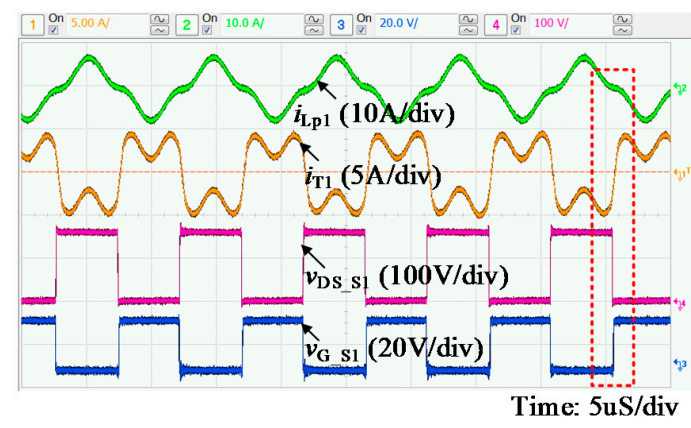

(a)

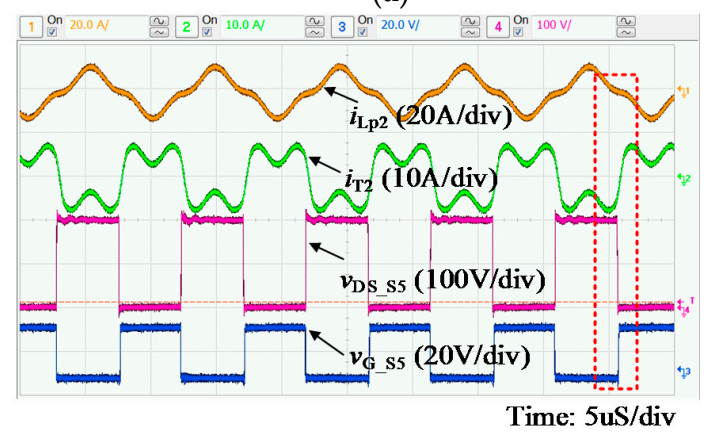

(c)

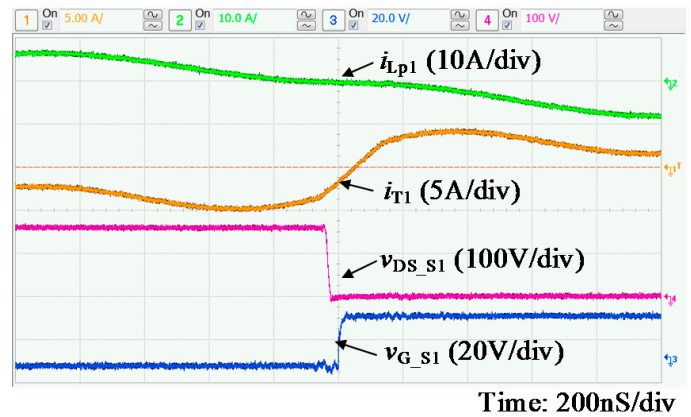

(b)

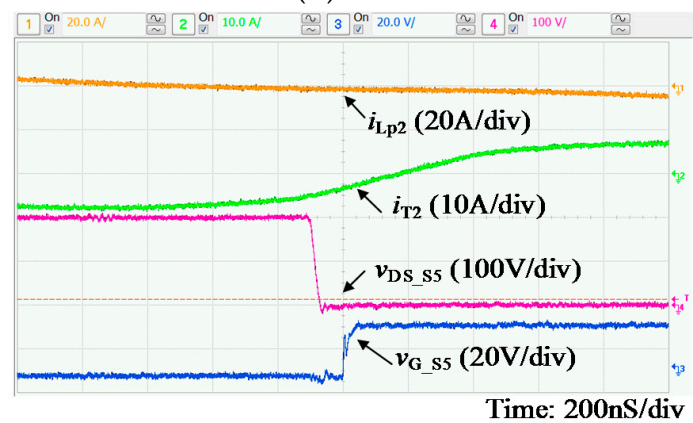

(d)

Figure 14. Cont. 


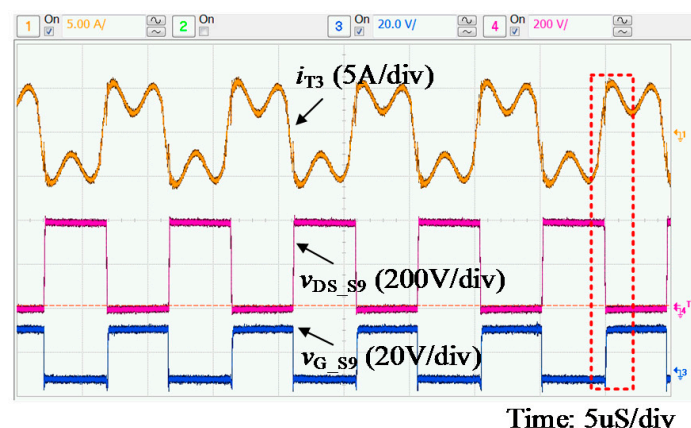

(e)

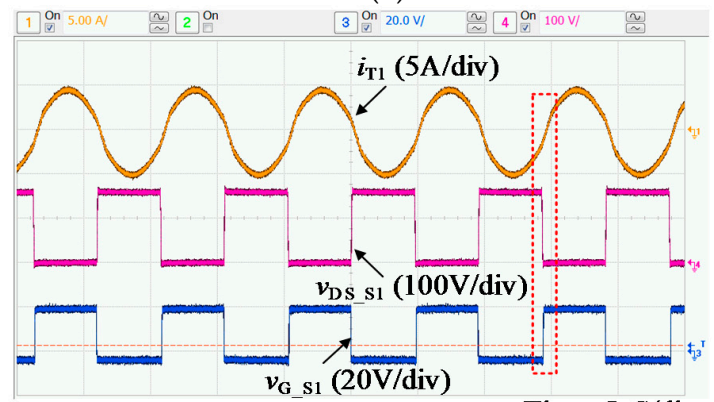

(g)

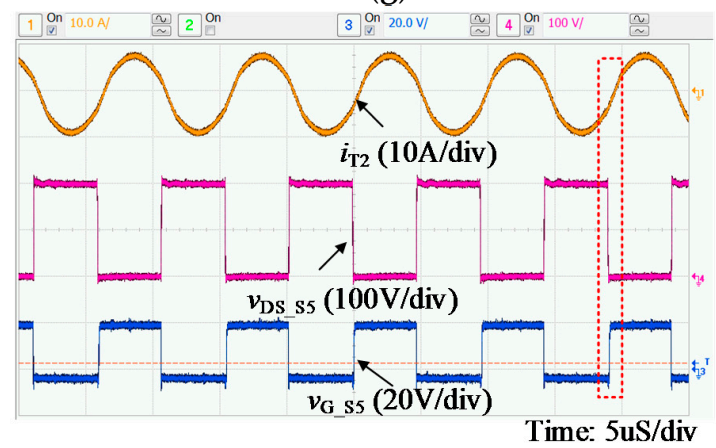

(i)

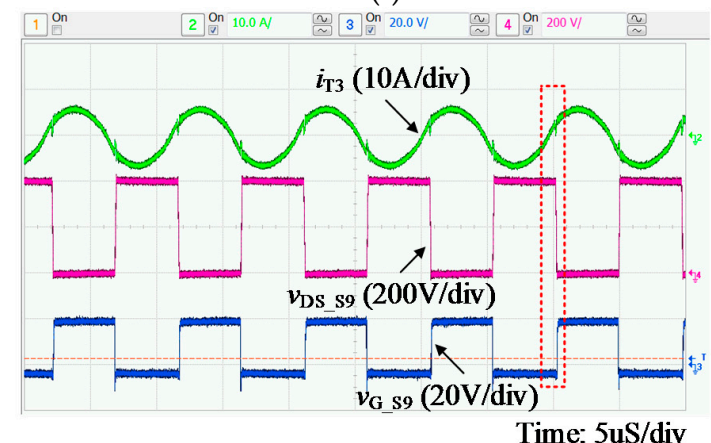

(k)

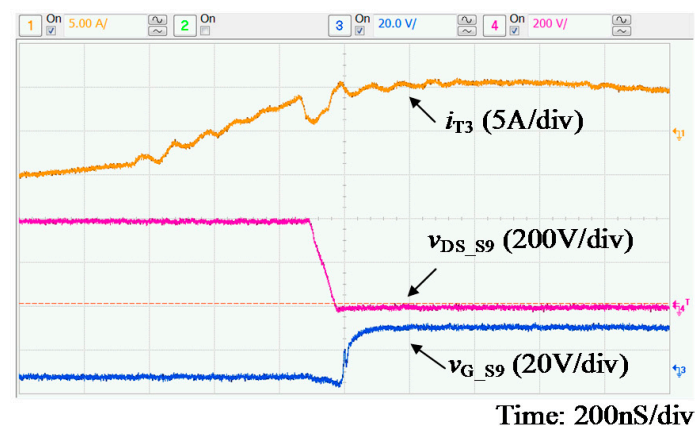

(f)

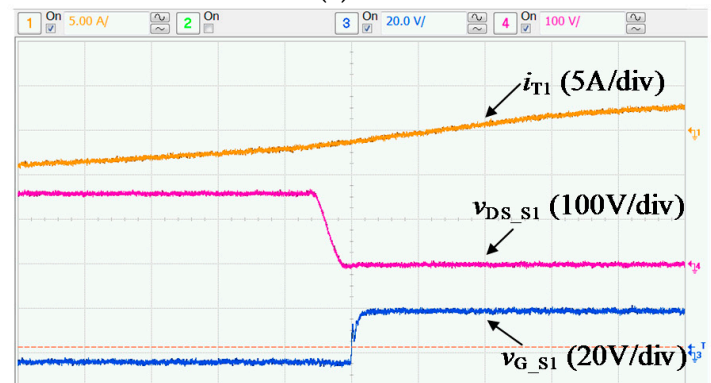

(h)

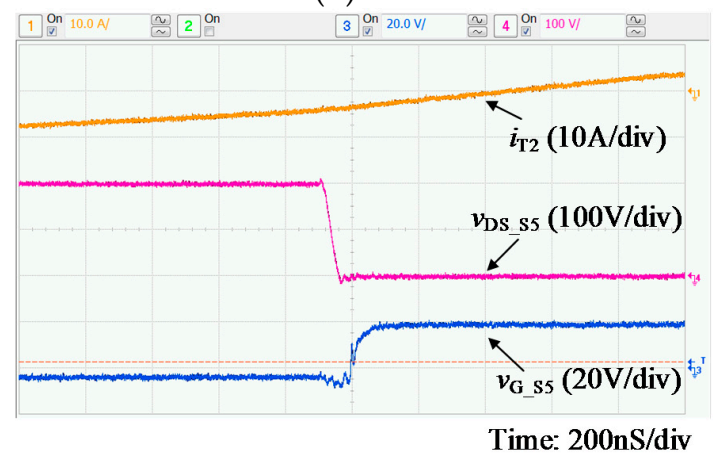

(j)

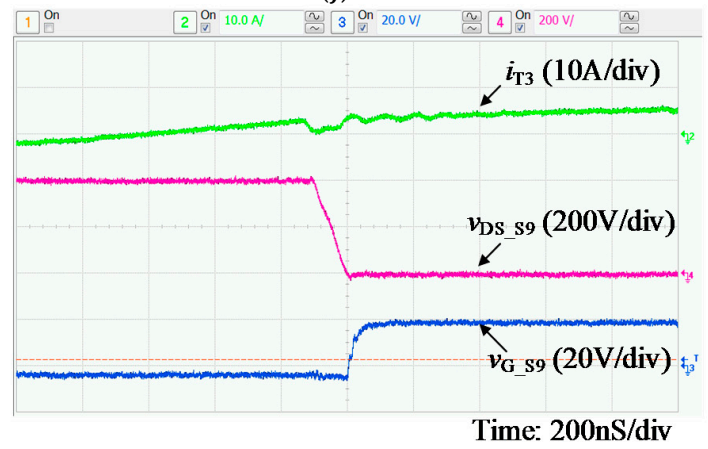

(1)

Figure 14. Experimental results of MR-TP-BDC and SR-TBC under rated condition: (a,b) bat; (c,d) sc $(\mathbf{e}, \mathbf{f})$ bus MR-TBC; (g,h) bat; $(\mathbf{i}, \mathbf{j})$ sc; $(\mathbf{k}, \mathbf{l})$ bus SR-TBC.

$i_{\mathrm{T} 1}, i_{\mathrm{T} 2}$ and $i_{\mathrm{T} 3}$ are the three-port currents of transformer. $i_{\mathrm{Lp} 1}$ and $i_{\mathrm{Lp} 2}$ are the parallel resonant inductance current. $v_{\mathrm{DS} \_} \mathrm{S} x$ represents the drain-source voltage of MOSFET $S_{x}(x$ is $1-9)$. $v_{\mathrm{G}_{-} S x}$ is the driving voltage of MOSFET $S_{x}(x$ is $1-9)$.

As the pictures show, like in the theoretical analysis, ZVS are realized for all switches in the three ports of MR-TBC and SR-TBC. Besides, the resonant currents are saddle-shaped in MR-TBC with the 3rd harmonic superposition while it presents sinusoidal waves in SR-TBC. The peaks of 
resonant currents are restricted with the 3rd harmonic function which are $7.87 \mathrm{~A}$ at SC port, $4.86 \mathrm{~A}$ at BAT port and 6.26 A at BUS port while they are 8.374 A at SC, $4.99 \mathrm{~A}$ at BAT and 6.78 A at BUS for SR-TBC. This will cause different losses in $P_{\text {tra }}$ and $P_{\mathrm{L}}$ as the analysis mentioned above.

Finally, the efficiency calculation and experimental results are summarized in Table 2, which indicate the precision of the theoretical calculation. The results also prove that MR-TBC displays better efficiency under the case of rating work.

Table 2. Efficiency comparison between MR-TBC and SR-TBC in $1500 \mathrm{~W}$.

\begin{tabular}{cccc}
\hline Prototype & Experiment Efficiency & Calculation Efficiency & Deviation \\
\hline MR-TBC 105 kHz & $95.45 \%$ & $96.36 \%$ & $0.91 \%$ \\
SR-TBC 105 kHz & $94.3 \%$ & $95.66 \%$ & $1.36 \%$ \\
\hline
\end{tabular}

(2) 1000 W Output Condition

In the $1000 \mathrm{~W}$ experiments, as the loss calculation, the working frequency is set to $110 \mathrm{kHz}$ for MR-TBC while $110 \mathrm{kHz}$ and $130 \mathrm{kHz}$ for SR-TBC. The current and voltage waveforms of BUS side of MR-TBC and SR-TBC are shown in Figure 15.

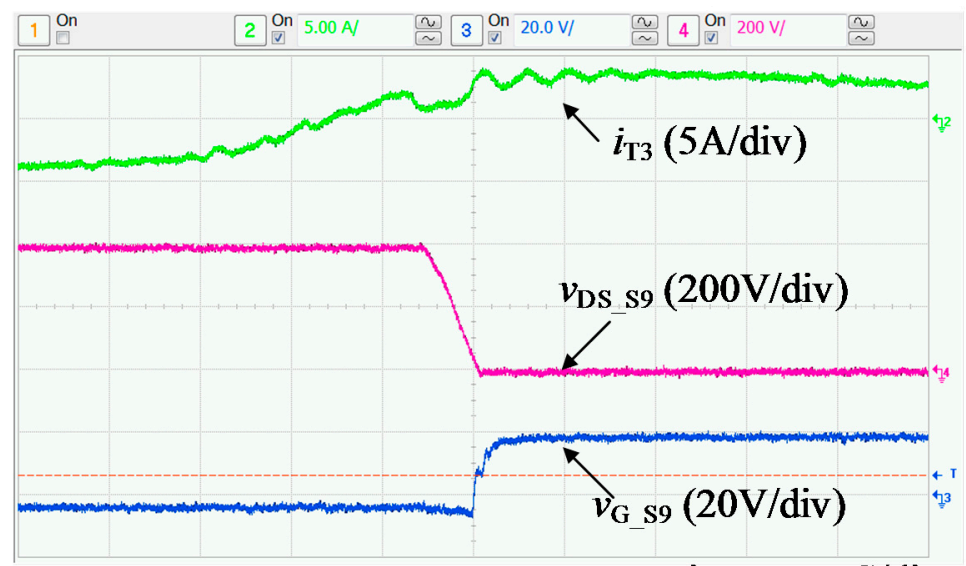

Time: $200 \mathrm{nS} / \mathrm{div}$

(a)

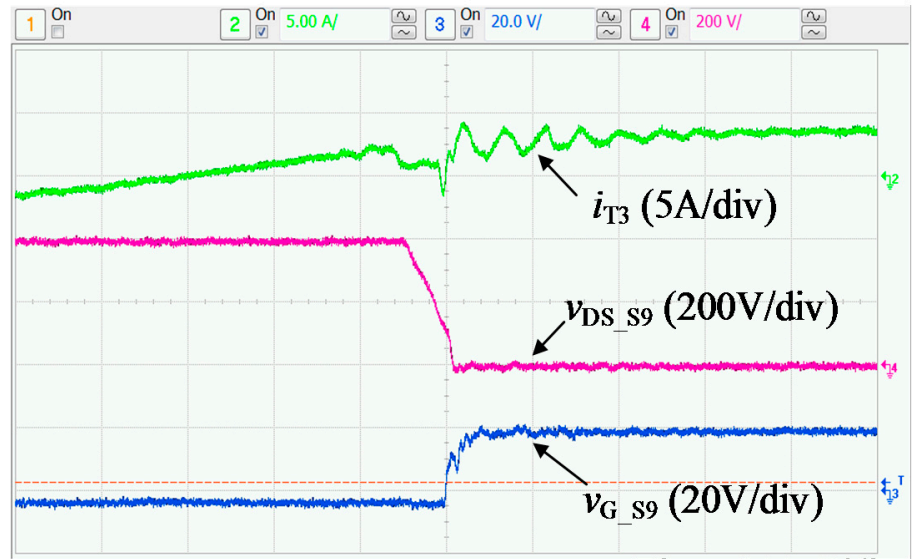

(b)

Time: $200 \mathrm{nS} / \mathrm{div}$

Figure 15. Cont. 


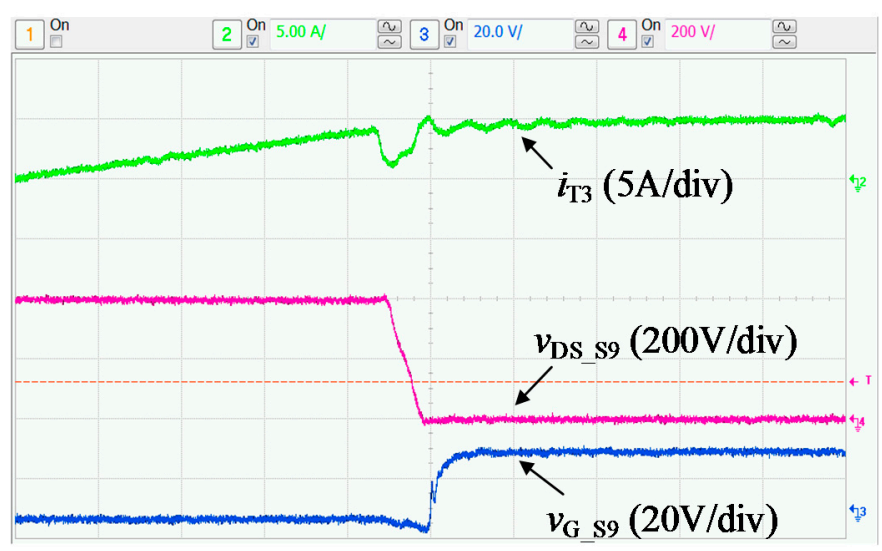

Time: $200 \mathrm{nS} / \mathrm{div}$

(c)

Figure 15. Experimental results of MR-TP-BDC and SR-TBC under 1000 W. (a) MR-TBC of $110 \mathrm{kHz}$; (b) SR-TBC of $110 \mathrm{kHz}$; (c) SR-TBC of $125 \mathrm{kHz}$.

As shown in Figure 15, when $f_{\text {sw }}$ is set to $110 \mathrm{kHz}$, the MR-TRC realizes ZVS while SR-TBC does not and causes current fluctuations. This makes the current change direction and leads to the turn-on loss $P_{\text {Sw_on }}$. On the other hand, when $f_{\mathrm{sw}}$ is set to $125 \mathrm{kHz}, \mathrm{ZVS}$ can be achieved by SR-TBC and the resonant is higher as the theoretical analysis. Also, for MR-TBC, the introduction of 3rd harmonic current reduces the peak current, $I_{\max }=4.17 \mathrm{~A}$, while it is $4.42 \mathrm{~A}$ for SR-TBC in $110 \mathrm{kHz}$ and $5.46 \mathrm{~A}$ in $125 \mathrm{kHz}$. Thus, $P_{\text {tra }}$ and $P_{\mathrm{L}}$ are relieved which is consistent with the calculation result. Finally, the efficiency calculation and experimental results are shown in Table 3.

Table 3. Efficiency comparison between MR-TBC and SR-TBC in $1000 \mathrm{~W}$.

\begin{tabular}{cccc}
\hline Prototype & Experiment Efficiency & Calculation Efficiency & Deviation \\
\hline MR-TBC $110 \mathrm{kHz}$ & $96.08 \%$ & $96.75 \%$ & $0.67 \%$ \\
SR-TBC $110 \mathrm{kHz}$ & $95.17 \%$ & $95.66 \%$ & $0.49 \%$ \\
SR-TBC $125 \mathrm{kHz}$ & $93 \%$ & $94.7 \%$ & $1.7 \%$ \\
\hline
\end{tabular}

\section{(3) 500 W Output Condition}

In case of $500 \mathrm{~W}$, considering the efficiency factor, the working frequency is set to $130 \mathrm{kHz}$ for MR-TBC and $130 \mathrm{kHz}$ and $150 \mathrm{kHz}$ for SR-TBC as references. The experiment current and voltage waveforms of BUS ports are shown in Figure 16.

As depicted in the picture, current fluctuations were observed in three cases, because the ZVS are not realized. However, in Figure 16a, the amplitude of current fluctuation is much smaller than the latter two cases. The cut-off voltage is also much less than the others. This makes the $P_{\text {sw_oss }_{-}}$and $P_{\text {sw_on }}$ to an acceptable range. On the other hand, a violent current fluctuation also generates adverse effects on transformers and inductors, resulting in extra loss. Finally, the efficiencies of the three groups are shown in Table 4.

Since the loss calculation does not consider the losses produced by PCB and parasitic parameters, the deviation of theoretical calculation and experiments can be accepted. From the Tables $2-4$, it can be found that the calculation deviation of SR-TBC is a little larger than for MR-TBC. This is because different resonant inductances are used in the two kinds of topology. This will produce a certain error in the process of making the inductance, which will affect the calculation results. 


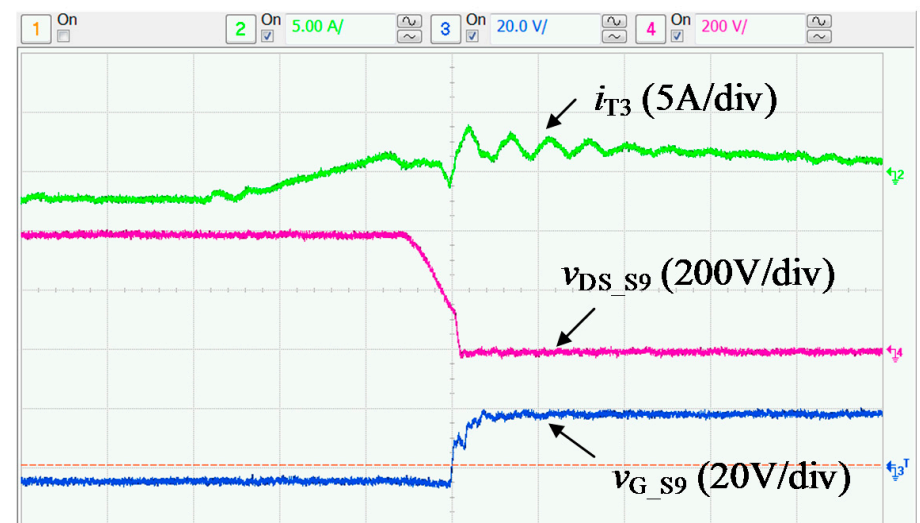

(a)

Time: $200 \mathrm{nS} / \mathrm{div}$

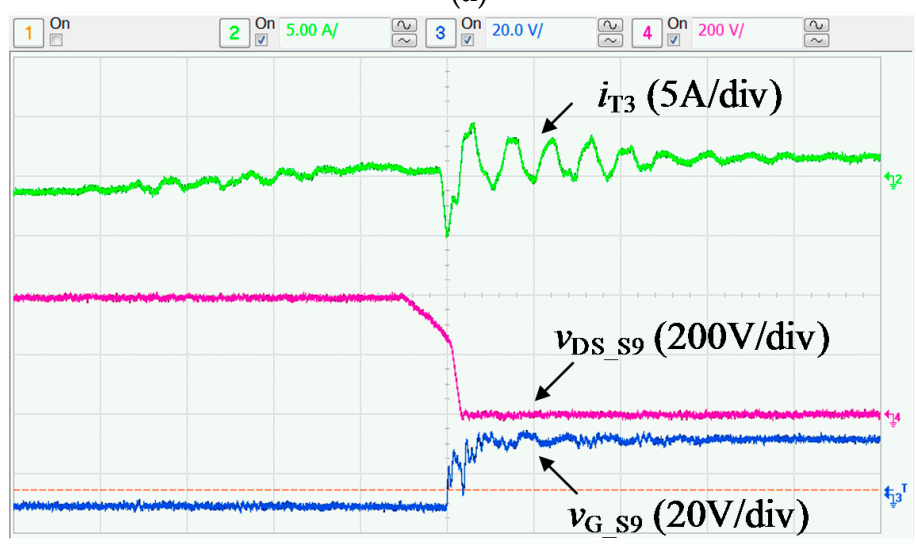

(b)

Time: $200 \mathrm{nS} / \mathrm{div}$

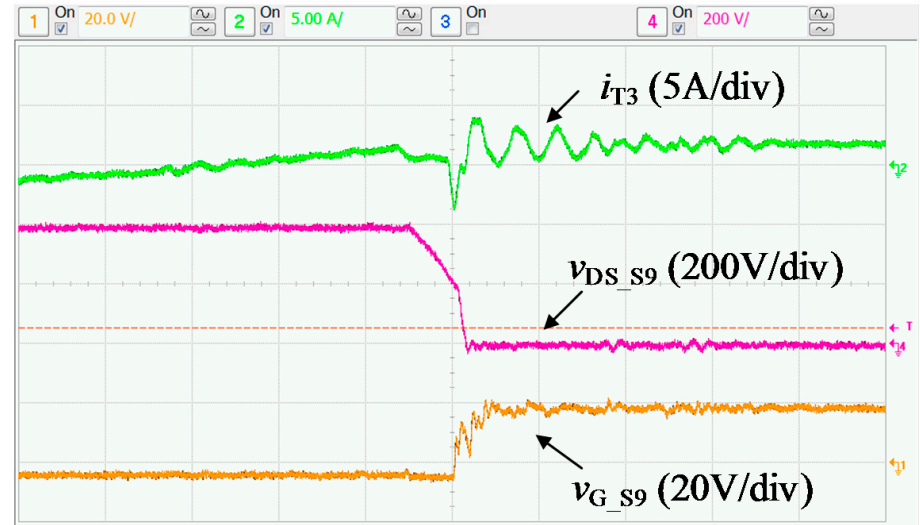

(c)

Time: $200 \mathrm{nS} / \mathrm{div}$

Figure 16. Experimental results of MR-TP-BDC and SR-TBC under $500 \mathrm{~W}$. (a) MR-TBC of $130 \mathrm{kHz}$; (b) SR-TBC of $130 \mathrm{kHz}$; (c) SR-TBC of $150 \mathrm{kHz}$.

Table 4. Efficiency comparison between MR-TBC and SR-TBC in $500 \mathrm{~W}$.

\begin{tabular}{cccc}
\hline Prototype & Experiment Efficiency & Calculation Efficiency & Deviation \\
\hline MR-TBC $130 \mathrm{kHz}$ & $94.45 \%$ & $95.14 \%$ & $0.69 \%$ \\
SR-TBC $130 \mathrm{kHz}$ & $91.67 \%$ & $92.82 \%$ & $1.15 \%$ \\
SR-TBC $150 \mathrm{kHz}$ & $93 \%$ & $93.52 \%$ & $0.52 \%$ \\
\hline
\end{tabular}


Based on the experiment results of MR-TBC and SR-TBC under 1500, 1000 and $500 \mathrm{~W}$, three-dimensional curves are drawn in Figure 17a reflecting the relations of the load, efficiency and frequency, as shown in Figure 17a. Since the reverse operation mode and the forward operation mode are similar in the loss distribution, the reverse three-dimensional surface curves can also be made as in Figure $17 \mathrm{~b}$. On the other hand, the frequency curves of ZVS realized can be drawn as in Figure 18a,b.

As the Figures 17 and 18 show, the MR-TBC has a wider ZVS range and higher work efficiency along the entire load range, both in the forward and reverse directions. Especially for the case of light load, the performance superiorities of MR-TBC are fully displayed.

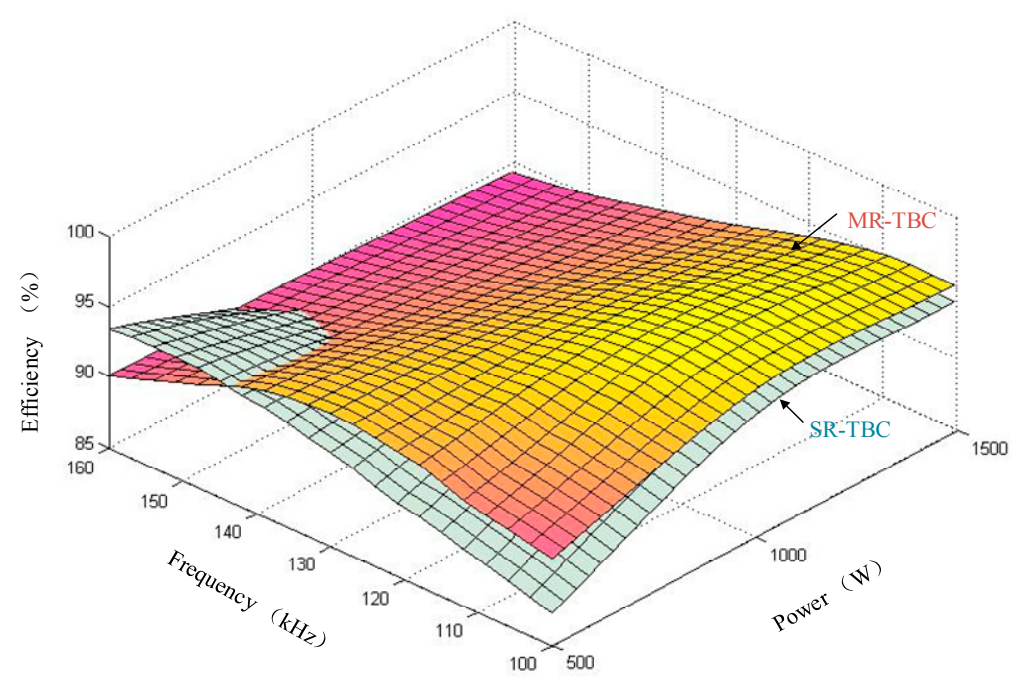

(a)

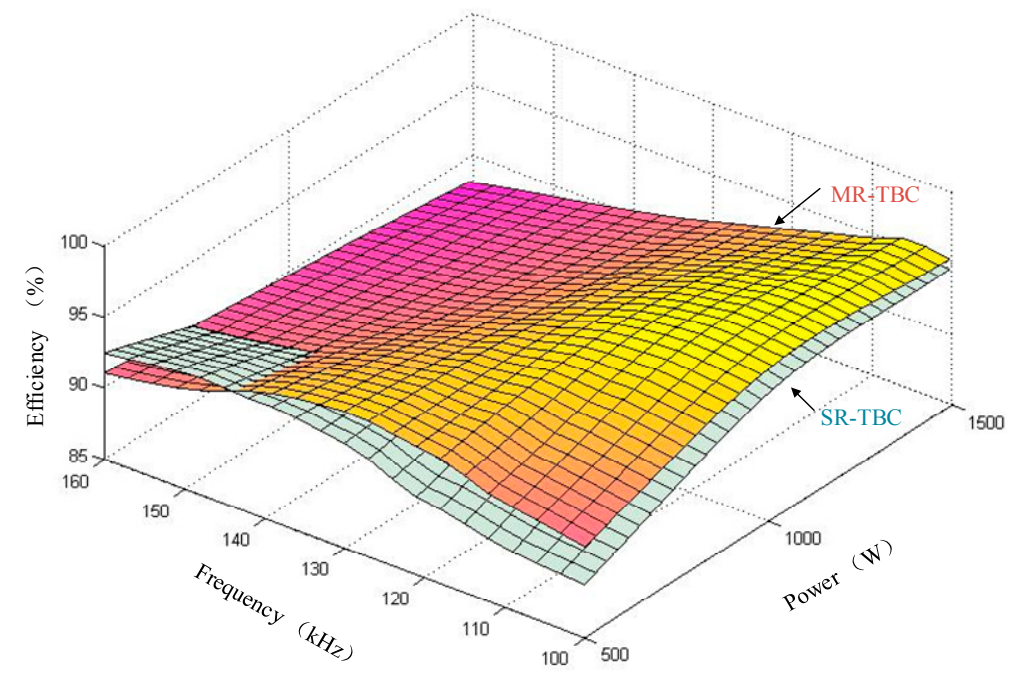

(b)

Figure 17. Efficiency three-dimensional graph of MR-TBC and SR-TBC (a) forward; (b) reverse. 


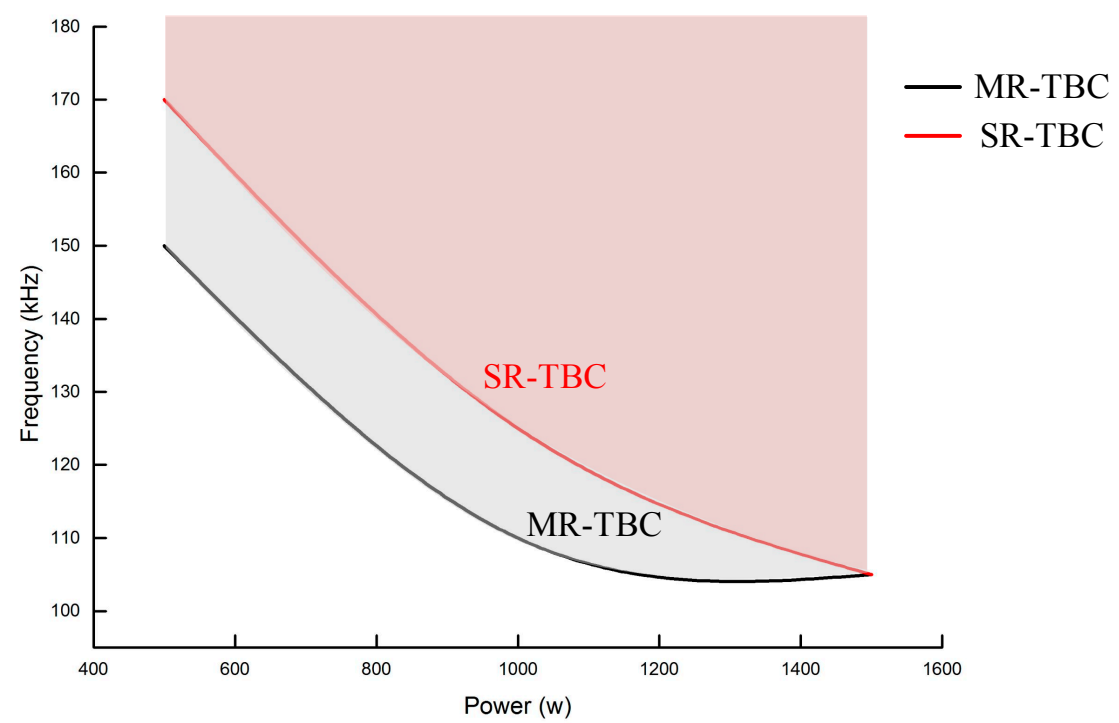

(a)

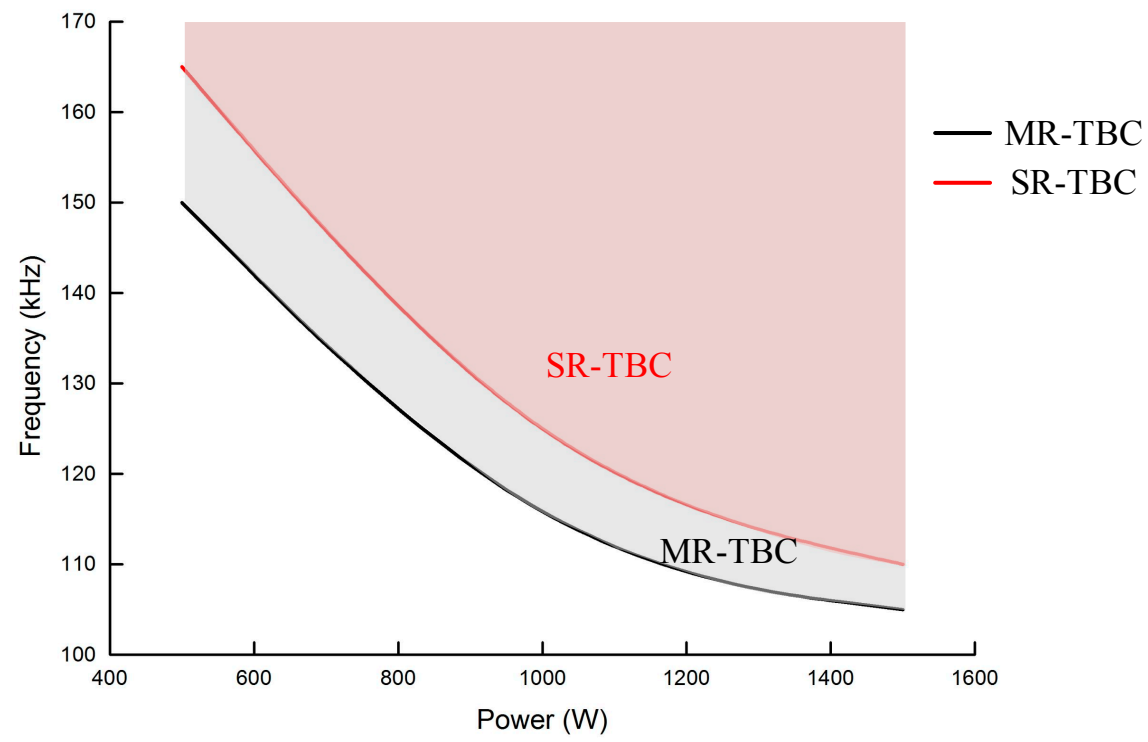

(b)

Figure 18. ZVS realized curve of MR-TBC and SR-TBC (a) forward; (b) reverse.

\section{Conclusions}

In this paper, a systematic loss analysis method is established for the MR-TBC and the SR-TBC. At the same time, a loss calculation between Si MOSFET and SiC MOSFET is discussed to verify that the SiC MOSFET can achieve the higher efficiency of $96.36 \%$ at rated power. It is because SiC MOSFET can avoid the free-wheeling diode turn-off loss. Also, some loss distributions are comparatively studied between SR-TBC and MR-TBC under different loads. The obtained results indicated that higher efficiency can be harvested in the full load range, and the broadened range of ZVS is achieved by the MR-TBC due to the transportation of 3rd harmonic current. In particular, under the light load conditions of the MR-TBC, the turn-on loss and output capacitor loss of MOSFET are limited to an acceptable range thanks to the quasi-ZVS characteristics. In addition, MR-TBC can decrease the peak current by introducing the 3rd harmonic current, which not only reduces the current stress but relieves the loss of magnet. As a result, the calculation efficiency is $95.14 \%$ for MR-TBC, while $93.12 \%$ for SR-TBC. In the end, relevant experiments at 500, 1000 and $1500 \mathrm{~W}$ are implemented to 
verify the accuracy of the theory. The results prove that the MR-TBC presents an evident advantage over the SR-TBC, since its efficiencies are relatively higher at the same power levels: $95.45 \%$ at $1500 \mathrm{~W}$, $96.08 \%$ at $1500 \mathrm{~W}$ and $94.45 \%$ at $500 \mathrm{~W}$.

Acknowledgments: This research was supported by the National High Technology Research and Development Program of China (863 Program) (Grant: 2015AA050603) and supported by Tianjin Municipal Science and Technology Commission (Grant: 14ZCZDGX00035). The authors would also like to thank the anonymous reviewers for their valuable comments and suggestions to improve the quality of the paper.

Author Contributions: Bo Chen, Ping Wang and Yifeng Wang designed the main parts of the study, including the circuit simulation model, loss model and experiment. Fuqiang Han and Wei Li helped in the hardware development, experiment and some theoretical analysis. Shuhuai Zhang helped in the experiment, related calculations and text retouching.

Conflicts of Interest: The authors declare no conflict of interest.

\section{References}

1. Kang, E.-C.; Lee, E.-J.; Ghorab, M.; Yang, L.; Entchev, E.; Lee, K.-S.; Lyu, N.-J. Investigation of energy and environmental potentials of a renewable trigeneration system in a residential application. Energies 2016, 9, 760. [CrossRef]

2. Pérez-Ortiz, M.; Jiménez-Fernández, S.; Gutiérrez, P.; Alexandre, E.; Hervás-Martínez, C.; Salcedo-Sanz, S. A review of classification problems and algorithms in renewable energy applications. Energies 2016, 9, 607. [CrossRef]

3. Kim, J.Y.; Kim, H.M.; Kim, S.K.; Jeon, J.H.; Choi, H.K. Designing an energy storage system fuzzy PID controller for microgrid islanded operation. Energies 2011, 4, 1443-1460. [CrossRef]

4. Hu, J.; Cheng, K.W.E. Predictive control of power electronics converters in renewable energy systems. Energies 2017, 10, 515. [CrossRef]

5. Xu, Q.; Hu, X.; Wang, P.; Xiao, J.; Tu, P.; Wen, C.; Lee, M.Y. A decentralized dynamic power sharing strategy for hybrid energy storage system in autonomous DC microgrid. IEEE Trans. Ind. Electron. 2016, 64, 5930-5941. [CrossRef]

6. Kollimalla, S.K.; Mishra, M.K.; Ukil, A.; Gooi, H.B. DC grid voltage regulation using new HESS control strategy. IEEE Trans. Sustain. Energy 2016, 8, 772-781. [CrossRef]

7. Zhang, N.; Sutanto, D.; Muttaqi, K.M. A review of topologies of three-port DC-DC converters for the integration of renewable energy and energy storage system. Renew. Sustain. Energy Rev. 2016, 56, 388-401. [CrossRef]

8. Zhu, H.; Zhang, D.; Zhang, B.; Zhou, Z. A nonisolated three-port dc-dc converter and three-domain control method for PV-battery power systems. IEEE Trans. Ind. Electron. 2015, 62, 4937-4947. [CrossRef]

9. Chien, L.J.; Chen, C.C.; Chen, J.F.; Hsieh, Y.-P. Novel three-port converter with high-voltage gain. IEEE Trans. Power Electron. 2014, 29, 4693-4703. [CrossRef]

10. Qian, Z.; Rahman, O.A.; Al-Atrash, H.; Batarseh, I. Modelling and control of three-port DC/DC converter interface for satellite applications. IEEE Trans. Power Electron. 2010, 25, 637-649. [CrossRef]

11. Wu, H.; Chen, R.; Zhang, J.; Xing, Y.; Hu, H.; Ge, H. A family of three-port half-bridge converters for a stand-alone renewable power system. IEEE Trans. Power Electron. 2011, 26, 2697-2706. [CrossRef]

12. Sun, X.; Shen, Y.; Li, W. A Novel LLC Integrated Three-port DC-DC converter for stand-alone PV/battery system. In Proceedings of the 2014 IEEE Conference and Expo Transportation Electrification Asia-Pacific (ITEC Asia-Pacific), Beijing, China, 31 August-3 September 2014; pp. 1-6.

13. Song, Y.; Wang, B. Survey on reliability of power electronic systems. IEEE Trans. Power Electron. 2013, 28, 591-604. [CrossRef]

14. Yang, S.; Bryant, A.; Mawby, P.; Xiang, D.; Ran, L.; Tavner, P. An industry-based survey of reliability in power electronic converters. IEEE Trans. Ind. Appl. 2009, 47, 1441-1451. [CrossRef]

15. Duarte, J.L.; Hendrix, M.; Simões, M.G. Three-port bidirectional converter for hybrid fuel cell systems. IEEE Trans. Power Electron. 2007, 22, 480-487. [CrossRef]

16. Fontana, C.; Buja, G.; Bertoluzzo, M.; Kumar, K.; Wang, Q. Power and control characteristics of an isolated three-port DC-DC converter under DCM operations. In Proceedings of the IECON 2016-42nd Annual Conference of the IEEE Industrial Electronics Society, Florence, Italy, 23-26 October 2016; pp. 4211-4216. 
17. Wang, L.; Wang, Z.; Li, H. Asymmetrical duty cycle control and decoupled power flow design of a three-port bidirectional DC-DC converter for fuel cell vehicle application. IEEE Trans. Power Electron. 2012, 27, 891-904. [CrossRef]

18. Ling, Z.; Wang, H.; Yan, K.; Gan, J. Optimal isolation control of three-port active converters as a combined charger for electric vehicles. Energies 2016, 9, 715. [CrossRef]

19. Phattanasak, M.; Gavagsaz-Ghoachani, R.; Martin, J.; Nahid-Mobarakeh, B.; Pierfederici, S.; Davat, B. Control of a hybrid energy source comprising a fuel cell and two storage devices using isolated three-port bidirectional DC-DC converters. IEEE Trans. Ind. Appl. 2015, 51, 491-497. [CrossRef]

20. Li, X.; Bhat, A.K.S. Analysis and design of high-frequency isolated duan-bridge series resonant DC/DC converter. IEEE Trans. Power Electron. 2010, 25, 850-862.

21. Krishnaswami, H.; Mohan, N. Three-port series-resonant DC-DC converter to interface renewable energy sources with bidirectional load and energy storage ports. IEEE Trans. Power Electron. 2010, 24, 2289-2297. [CrossRef]

22. Shreelekha, K.; Arulmozhi, S. Multiport isolated bidirectional DC-DC converter interfacing battery and supercapacitor for hybrid energy storage application. In Proceedings of the International Conference on Electrical, Electronics and Optimization Techiniques, Chennai, India, 3-5 March 2016; pp. 2763-2768.

23. Wang, C.-S.; Li, W.; Wang, Y.-F.; Chen, B. A high-efficiency isolated LCLC multi-resonant three-port bidirectional DC-DC converter. Energies 2017, 10, 934. [CrossRef]

24. Zhou, Y.; Huang, W.; Hong, F.; Wang, C. Modelling analysis and power loss of coupled-inductor single-stage boost inverter based grid-connected photovoltaic power system. IET Power Electron. 2016, 9, 1664-1674. [CrossRef]

25. Wang, X.; Jiang, C.; Lei, B.; Teng, H.; Bai, H.K.; Kirtley, J.L. Power-loss analysis and efficiency maximization of a silicon-carbide MOSFET-based three-phase 10-kW bidirectional EV charger using variable-DC-bus control. IEEE J. Emerg. Sel. Top. Power Electron. 2016, 4, 880-892. [CrossRef]

26. Fatemi, A.; Azizi, M.; Mohamadian, M.; Varjani, A.Y. Single-phase dual-output inverters with three-switch legs. IEEE Trans. Ind. Electron. 2013, 60, 1769-1779. [CrossRef]

27. Meng, Z.; Wang, Y.-F.; Yang, L.; Li, W. Analysis of power loss and improved simulation method of a high frequency dual-buck full-bridge inverter. Energies 2017, 10, 311. [CrossRef]

28. Bonislawski, M.; Holub, M. Averaged inverter loss estimation algorithm. In Proceedings of the 2016 18th European Conference on Power Electronics and Applications, Karlsruhe, Germany, 5-9 September 2016; pp. 1-9.

29. Tanimoto, Y.; Saito, A.; Matsuura, K.; Kikuchihara, H. Power-loss prediction of high-voltage SiC-MOSFET, circuits with compact model including carrier-trap influences. IEEE Trans. Power Electron. 2016, 31, 4509-4516. [CrossRef]

30. Wang, J.; Zhao, T.; Li, J.; Huang, A.Q. Characterization, modeling, and Application of 10-kV SiC MOSFET. IEEE Trans. Electron Devices 2008, 55, 1798-1806. [CrossRef]

31. Guan, Y.; Wang, Y.; Xu, D.; Wang, W. A 1 MHz half-bridge resonant DC/DC converter based on GaN FETs and planar magnetics. IEEE Trans. Power Electron. 2017, 32, 2876-2891. [CrossRef]

32. Dowell, P.L. Effects of eddy currents in transformer windings. Proc. Inst. Electr. Eng. 1966, 113, 1387-1394. [CrossRef]

33. Ferreira, J.A. Improved analytical modeling of conductive losses in magnetic components. IEEE Trans. Power Electron. 1994, 9, 127-131. [CrossRef]

(C) 2017 by the authors. Licensee MDPI, Basel, Switzerland. This article is an open access article distributed under the terms and conditions of the Creative Commons Attribution (CC BY) license (http://creativecommons.org/licenses/by/4.0/). 